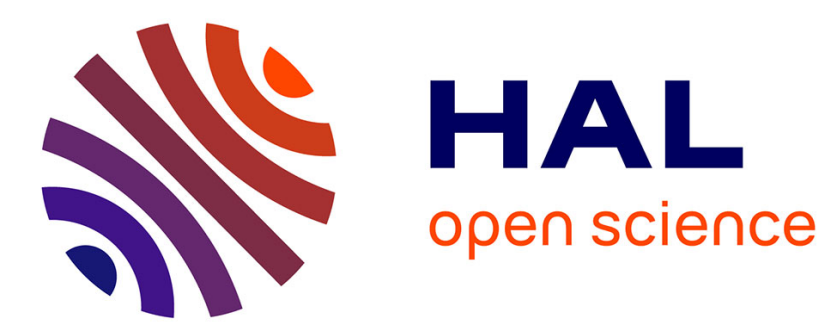

\title{
Functional convergence of quantile-type frontiers with application to parametric approximations
}

\author{
Abdelaati Daouia, Jean-Pierre Florens, Léopold Simar
}

\section{To cite this version:}

Abdelaati Daouia, Jean-Pierre Florens, Léopold Simar. Functional convergence of quantile-type frontiers with application to parametric approximations. Journal of Statistical Planning and Inference, 2008, 138 (3), pp.708-725. 10.1016/j.jspi.2007.01.005 . hal-02660040

\section{HAL Id: hal-02660040 \\ https://hal.inrae.fr/hal-02660040}

Submitted on 30 May 2020

HAL is a multi-disciplinary open access archive for the deposit and dissemination of scientific research documents, whether they are published or not. The documents may come from teaching and research institutions in France or abroad, or from public or private research centers.
L'archive ouverte pluridisciplinaire HAL, est destinée au dépôt et à la diffusion de documents scientifiques de niveau recherche, publiés ou non, émanant des établissements d'enseignement et de recherche français ou étrangers, des laboratoires publics ou privés. 


\title{
Functional Convergence of Quantile-type Frontiers with Application to Parametric Approximations
}

\author{
AbdelaAti Daouia \\ Université de Toulouse I, GREMAQ, France \\ daouia@cict.fr \\ JEAN-PIERRE FLORENS \\ Université de Toulouse I, GREMAQ, France \\ florens@cict.fr \\ LÉOPOLD SIMAR* \\ Université Catholique de Louvain, Institut de Statistique, Belgium \\ simar@stat.ucl.ac.be
}

Revision, June 8, 2006

\begin{abstract}
Nonparametric estimators of the upper boundary of the support of a multivariate distribution are very appealing because they rely on very few assumptions. But in productivity and efficiency analysis, this upper boundary is a production (or a cost) frontier and a parametric form for it allows for a richer economic interpretation of the production process under analysis. On the other hand, most of the parametric approaches rely on often too restrictive assumptions on the stochastic part of the model and are based on standard regression techniques fitting the shape of the center of the cloud of points rather than its boundary. To overcome these limitations, Florens and Simar (2005) propose a two-stage approach which tries to capture the shape of the cloud of points near its frontier by providing parametric approximations of a nonparametric frontier. In this paper we propose an alternative method using the nonparametric quantile-type frontiers introduced in Aragon, Daouia and Thomas-Agnan (2005) for the nonparametric part of our model. These quantile-type frontiers have the superiority of being more robust to extremes. Our main result concerns the functional convergence of the quantile-type frontier process. Then we provide convergence and asymptotic normality of the resulting estimators of the parametric approximation. The approach is illustrated through simulated and real data sets.
\end{abstract}

Key words : Functional convergence; Nonstandard conditional quantile-type frontiers; Parametric approximations of nonparametric estimators.

${ }^{*}$ Corresponding Author: Institut de Statistique, 20 voie du roman pays, B-1348 Louvain-la-Neuve, Belgium. Tel: +32 10 474308, Fax: +32 10 473032. Research support from the "Interuniversity Attraction Pole", Phase V (No. P5/24) from the Belgian Science Policy is also acknowledged. 


\section{Basic Concepts and Notations}

Let $\Psi$ be the support of the joint distribution of a random vector $(X, Y) \in \mathbb{R}_{+}^{p} \times \mathbb{R}_{+}$defined on the probability space $(\Omega, \mathcal{A}, P)$. Consider the problem of estimating the upper boundary of $\Psi$ from a sample $\left\{\left(X_{1}, Y_{1}\right), \cdots,\left(X_{n}, Y_{n}\right)\right\}$ of independent random vectors with the same distribution as $(X, Y)$. If $\mathcal{X}=\left\{x \in \mathbb{R}_{+}^{p} \mid Y(x) \neq \emptyset\right\}$ where $Y(x)=\left\{y \in \mathbb{R}_{+} \mid(x, y) \in \Psi\right\}$, then define $\varphi(x)=\max \{y: y \in Y(x)\}$ for any $x \in \mathcal{X}$. The upper boundary of $\Psi$ can be then defined as the set $\{(x, \varphi(x)) \mid x \in \mathcal{X}\}$. This boundary is assumed to be nondecreasing in the sense that the frontier function $\varphi(x)$ is nondecreasing ${ }^{1}$ in $x$.

This kind of problem appears naturally to be useful when analyzing production performance of firms, where $X$ represents the vector of inputs (resources of production) and $Y$ is the output (a quantity of produced goods). In this context, $\varphi(x)$ is the production frontier, i.e., the maximal achievable level of output for a firm working at the level of inputs $x$. The production efficiency of a firm operating at the level $(x, y)$ can be then measured by the relative comparison of its output $y$ with the reference frontier $\varphi(x)$. These methods have been widely applied to examine efficiency in a variety of industries; see Lovell (1993) and Seiford (1996) for comprehensive bibliographies of these applications. Aside from the production setting, the problem of estimating monotone boundaries also naturally occurs in portfolio management. In capital asset pricing models (CAPM), the objective is to analyze the performance of investment portfolios. Risk and average return on a portfolio are analogous to inputs and outputs in models of production; in CAPM, the boundary of the attainable set of portfolios gives a benchmark relative to which the efficiency of a portfolio can be measured. These models were developed by Markovitz (1959) and others; Sengupta (1991) and Sengupta and Park (1993) provide links between CAPM and nonparametric estimation of frontiers.

We will follow the probabilistic formulation of the frontier problem proposed by Cazals, Florens and Simar (2002, CFS hereafter). Let us denote by $F(y \mid x)=F(x, y) / F_{X}(x)$ the nonstandard $^{2}$ conditional distribution function of $Y$ given $X \leq x$, where $F$ is the joint distribution function of $(X, Y)$ and $F_{X}(x)=F(x, \infty)$. From now on we assume that $x \in \mathbb{R}_{+}^{p}$ is such that $F_{X}(x)>0$. The monotone upper surface of $\Psi$ can be then characterized through the graph of the frontier function

$$
\varphi(x)=\inf \left\{y \in \mathbb{R}_{+} \mid F(y \mid x)=1\right\} .
$$

\footnotetext{
${ }^{1}$ For $x$ and $x^{\prime}$ in $\mathbb{R}^{p}$ the inequality $x \leq x^{\prime}$ has to be understood componentwise. A real valued function $r$ on $\mathbb{R}^{p}$ is then said to be nondecreasing with respect to this partial order if $x \leq x^{\prime}$ implies $r(x) \leq r\left(x^{\prime}\right)$.

${ }^{2}$ We use the word "nonstandard" to focus on the unusual condition $X \leq x$ in place of the more common $X=x$.
} 
This value represents the upper boundary of the support of the nonstandard conditional probability measure of $Y$ given $X \leq x$.

In our approach, we focus on a deterministic frontier model where the support $\Psi$ contains all the observations $\left(X_{i}, Y_{i}\right)$ with probability 1 . Parametric approaches have been first proposed in the econometric literature (this includes, among others, Aigner and Chu, 1968 and Greene, 1980). Here we assume the frontier function $\varphi(x)$ can be written as a specified analytical function $\varphi_{\theta}(x)$ depending on a finite number of parameters $\theta \in \mathbb{R}^{k}$. We denote this parametric model by

$$
Y_{i}=\varphi_{\theta}\left(X_{i}\right)-U_{i} \quad \text { with } \quad U_{i} \geq 0 \quad \text { a.s. }
$$

If we specify an appropriate stochastic model for the error terms $U_{i}$, Greene (1980) proposes estimators of $\theta$ based on Ordinary Least Squares (shifted- or corrected-OLS to take into account for the positiveness of the error term) or on maximum likelihood methods. In these approaches, $U_{i}$ are i.i.d. random variables, supposed to be independent of $X_{i}$, so that from (1.1) we have $\varphi_{\theta}\left(X_{i}\right)=E\left(Y_{i} \mid X_{i}\right)+\mu_{U}$ where $\mu_{U}=E\left(U_{i}\right)$.

On one hand parametric models are very appealing because the parameters are easy to estimate and to interpret (in terms of elasticities or returns to scale,...) but on the other hand their properties rely on restrictive assumptions on the stochastic part of the model (parametric specification of the law of $U$, homoscedasticity,...) and they are based on standard regression tools which capture the shape of the cloud of points $\left\{\left(X_{1}, Y_{1}\right), \cdots,\left(X_{n}, Y_{n}\right)\right\}$ near its center rater than near its optimal boundary, because, at a constant shift $\mu_{U}, \varphi_{\theta}(X)$ is the regression function of $Y$ on $X$ (see Florens and Simar, 2005 for a careful discussion).

To overcome these drawbacks, Simar (1992) proposed (without analyzing the statistical properties of the obtained estimators) to identify in a first step, where is located the upper frontier by using a nonparametric method, then in a second step, to adjust the parametric model to the obtained nonparametric frontier. From an economic point of view, the first step can be viewed as a kind of "filtering" for eliminating from the sample clearly inefficient units which certainly do not provide substantial information to analyse how to transform efficiently inputs into output.

We can use in this first step filtering the flexible nonparametric Free Disposal Hull (FDH) estimator (Deprins, Simar and Tulkens, 1984),

$$
\widehat{\varphi}_{n}(x)=\inf \left\{y \in \mathbb{R}_{+} \mid \widehat{F}_{n}(y \mid x)=1\right\}=\max _{i \mid X_{i} \leq x} Y_{i}
$$

where $\widehat{F}_{n}(y \mid x)=\widehat{F}_{n}(x, y) / \widehat{F}_{X, n}(x)$, with $\widehat{F}_{n}(x, y)=(1 / n) \sum_{i=1}^{n} 1\left(X_{i} \leq x, Y_{i} \leq y\right)$ and $\widehat{F}_{X, n}(x)=\widehat{F}_{n}(x, \infty)$. The asymptotic of $\widehat{\varphi}_{n}(x)$ was first derived by Korostelev, Simar and 
Tsybakov (1995) for the consistency and by Park, Simar and Weiner (2000) for the asymptotic sampling distribution. The drawback of the FDH estimator lies in the fact that it is, by construction, very sensitive to extreme values. CFS have proposed alternatives which are shown to be more robust than the FDH estimator. They are based on the concept of partial order- $m$ expected frontiers defined as $\varphi_{m}(x)=E\left(\max \left(Y^{1}, \ldots, Y^{m}\right) \mid X \leq x\right)$, where for an integer $m \geq 1,\left(Y^{1}, \ldots, Y^{m}\right)$ are $m$ independent identically distributed random variables generated by the distribution of $Y$ given $X \leq x$. It turns out that

$$
\varphi_{m}(x)=\int_{0}^{\varphi(x)}\left(1-[F(y \mid x)]^{m}\right) d y,
$$

which can be nonparametrically estimated by

$$
\widehat{\varphi}_{m, n}(x)=\int_{0}^{\widehat{\varphi}_{n}(x)}\left(1-\left[\widehat{F}_{n}(y \mid x)\right]^{m}\right) d y
$$

The order $m$ can be viewed as a trimming parameter which tunes the robustness of the estimator since, as pointed in CFS, when $m \rightarrow \infty, \varphi_{m}(x)$ converges to the full frontier $\varphi(x)$ and $\widehat{\varphi}_{m, n}(x)$ converges to the FDH estimator $\widehat{\varphi}_{n}(x)$.

Florens and Simar (2005) provide the asymptotic properties of the resulting "semiparametric" estimators of $\theta$ when these two nonparametric estimators of the frontier are used in the first "filtering" stage. In this paper we propose an alternative method using the nonparametric quantile-type frontiers introduced in Aragon, Daouia and Thomas-Agnan (2005). The interest is that these quantile-type estimators are more resistant to extremes than the CFS order- $m$ estimators as established in Daouia and Ruiz-Gazen (2004) from a robustness theory point of view (qualitative robustness and influence function). See also Daouia and Simar (2006) for theoretical robustness properties in a full multivariate setup.

Similarly to the order- $m$ partial frontier, the order- $\alpha$ partial frontier function increases w.r.t. the continuous order $\alpha \in[0,1]$ and converges to the full frontier $\varphi(x)$ as $\alpha \nearrow 1$. It is defined, for a given level $x$, by the $\alpha$-quantile of the distribution of $Y$ given $X \leq x$,

$$
q_{\alpha}(x):=F^{-1}(\alpha \mid x)=\inf \left\{y \in \mathbb{R}_{+} \mid F(y \mid x) \geq \alpha\right\}
$$

A simple estimator of $q_{\alpha}(x)$, which increases and converges to the $\mathrm{FDH} \widehat{\varphi}_{n}(x)$ as $\alpha \nearrow 1$, is easily derived by inverting the empirical version $\widehat{F}_{n}(y \mid x)$ of $F(y \mid x)$,

$$
\widehat{q}_{\alpha, n}(x):=\widehat{F}_{n}^{-1}(\alpha \mid x)=\inf \left\{y \in \mathbb{R}_{+} \mid \widehat{F}_{n}(y \mid x) \geq \alpha\right\}
$$

This estimator is very fast to compute, and the value of the tunning parameter $\alpha$ is easy to interpret (for a unit located on the $\alpha$-quantile frontier $\left(x, q_{\alpha}(x)\right.$ ), there is a probability $1-\alpha$ of being dominated by firms using less inputs than the level $x$ ). The nonparametric 
estimator satisfies very similar statistical properties to those of the CFS estimator. In summary, it converges at the rate $\sqrt{n}$, it is asymptotically unbiased and normally distributed. Moreover, when the order $\alpha$ is considered as a function of $n$ which tends to one as $n \rightarrow \infty$, $\widehat{q}_{\alpha(n), n}(x)$ estimates the true frontier function $\varphi(x)$ and shares the same asymptotic distribution (Weibull) of the FDH estimator $\widehat{\varphi}_{n}(x)$, as described in Park et al. (2000), provided that $n^{(p+2) /(p+1)}(1-\alpha(n)) \rightarrow 0$, but for finite $n$, the estimator will not envelop all the data points and so is more robust to extremes and outliers.

The paper is organized as follows. Section 2 extends previous results of convergence of $\widehat{q}_{\alpha, n}(x)$, by establishing the functional convergence of $\sqrt{n}\left(\widehat{q}_{\alpha, n}-q_{\alpha}\right)$ as being a process indexed by $x$. Section 3 proposes then the parametric approximations of the quantile frontier and develops the asymptotic distribution of the resulting estimators, for linear parametric models as well as for general parametric models. It also provides new results for estimating the parametric model for the full frontier itself, by using the more robust order- $m$ or order$\alpha$ frontiers in the first step. It establishes the convergence at an exponential rate of the estimators of the parametric models for the true frontier, by chosing $m$ and $\alpha$ as appropriate functions of $n$. Section 4 illustrates and compare the different approaches with simulated and real data sets. Section 5 concludes. All the proofs are reported in the appendix.

\section{Functional Convergence Theorem}

Let $x$ be a fixed value such that $F_{X}(x)>0$. Assume that the conditional distribution function $F(\cdot \mid x)$ is differentiable at $q_{\alpha}(x)$ with derivative $f\left(q_{\alpha}(x) \mid x\right)>0$. Aragon et al. (2005) have proved

$$
\widehat{q}_{\alpha, n}(x)-q_{\alpha}(x)=\frac{\alpha \widehat{F}_{X, n}(x)-\widehat{F}_{n}\left(x, q_{\alpha}(x)\right)}{f\left(q_{\alpha}(x) \mid x\right) F_{X}(x)}+R_{n}^{\alpha}(x)
$$

where the remainder term $R_{n}^{\alpha}(x)$ becomes negligible as $n \rightarrow \infty$. More precisely, they have shown $\sqrt{n} R_{n}^{\alpha}(x)=o_{p}(1)$ as $n \rightarrow \infty$. This result has been improved in Daouia (2005) who extends (2.1) to a Bahadur-type asymptotic representation, namely $R_{n}^{\alpha}(x)=$ $O\left(n^{-3 / 4}(\log n)^{1 / 2}(\log \log n)^{1 / 4}\right)$ as $n \rightarrow \infty$, with probability 1 . He also proves the functional convergence of $\sqrt{n}\left(\widehat{q}_{\alpha, n}(x)-q_{\alpha}(x)\right)$ as a process indexed by the order $\alpha$ in the space $L^{\infty}(] 0,1[)$ of bounded functions on ]0,1[. However, Daouia (2005) raises the question of finding the asymptotic limit of $\sqrt{n}\left(\widehat{q}_{\alpha, n}(x)-q_{\alpha}(x)\right)$ as a process indexed by $x \in \mathbb{R}^{p}$. This issue is addressed in the next theorem.

Throughout this section the order $\alpha$ is arbitrarily fixed in $] 0,1\left[\right.$ and $K \subset \mathbb{R}_{+}^{p}$ is an arbitrarily fixed set such that $\inf _{x \in K} F_{X}(x)>0$. The support of $Y$ is assumed to have a finite upper boundary. 
Theorem 2.1. Assume that $F(\cdot \mid x)$ is differentiable for any $x \in K$, with derivative $f(\cdot \mid x)$ such that $\sup _{(x, y) \in K \times \mathbb{R}_{+}} f(y \mid x)<\infty, \inf _{x \in K} f\left(q_{\alpha}(x) \mid x\right)>0$ and the differentiability of $F(\cdot \mid x)$ at $q_{\alpha}(x)$ is uniform in $x \in K$. If furthermore $F$ is continuous, then the conditional empirical quantile process $\sqrt{n}\left(\widehat{q}_{\alpha, n}-q_{\alpha}\right)$, indexed by $x \in K$, converges in distribution in $L^{\infty}(K)$ to a centered Gaussian process with covariance function defined, for any $x^{1}, x^{2} \in K$, by

$$
\begin{aligned}
& \Gamma_{\alpha}\left(x^{1}, x^{2}\right)=\left\{\left[f\left(q_{\alpha}\left(x^{1}\right) \mid x^{1}\right) F_{X}\left(x^{1}\right)\right]\left[f\left(q_{\alpha}\left(x^{2}\right) \mid x^{2}\right) F_{X}\left(x^{2}\right)\right]\right\}^{-1} \times \\
& \left\{\alpha^{2} F_{X}\left(x^{1} \wedge x^{2}\right)-\alpha F\left(x^{1} \wedge x^{2}, q_{\alpha}\left(x^{2}\right)\right)-\alpha F\left(x^{1} \wedge x^{2}, q_{\alpha}\left(x^{1}\right)\right)+F\left(x^{1} \wedge x^{2}, q_{\alpha}\left(x^{1}\right) \wedge q_{\alpha}\left(x^{2}\right)\right)\right\} .
\end{aligned}
$$

Theorem 2.1 and the following corollary will serve our purposes to prove the asymptotic normality of our semi-parametric estimators described in Section 3.

Corollary 2.1. Under the conditions of Theorem 2.1, we have

$$
\sup _{x \in K} \sqrt{n}\left|R_{n}^{\alpha}(x)\right| \stackrel{P}{\longrightarrow} 0 \quad \text { as } \quad n \rightarrow \infty
$$

\section{Parametric Approximations of Frontiers}

Throughout this section $\mu$ is a finite positive measure on the support of $X$. Consider a parametric family of functions $\varphi_{\theta}(x)$ defined on $\mathbb{R}_{+}^{p}$ and depending on a finite number of parameters $\theta \in \mathbb{R}^{k}$. We will consider two parametric approximations: one for the order- $\alpha$ frontier function $q_{\alpha}$ and one for the full frontier function $\varphi$. The best parametric approximation of the true frontier function $\varphi$ in the parametric family $\left\{\varphi_{\theta} \mid \theta \in \mathbb{R}^{k}\right\}$, in the $L^{2}(K, \mu)$ norm, is defined through the pseudo-true value of $\theta$ :

$$
\theta(K, \mu)=\arg \min _{\theta \in \mathbb{R}^{k}} \int_{K}\left(\varphi(x)-\varphi_{\theta}(x)\right)^{2} d \mu(x) .
$$

If model (1.1) is true, this coincides with the true value $\theta$. A weakly consistent estimator of $\theta(K, \mu)$ has been suggested in Florens and Simar (2005). It is defined as follows:

$$
\widehat{\theta}_{n}(K, \mu)=\arg \min _{\theta \in \mathbb{R}^{k}} \int_{K}\left(\widehat{\varphi}_{n}(x)-\varphi_{\theta}(x)\right)^{2} d \mu(x) .
$$

But this estimator suffers from the dramatic lack of robustness of the FDH frontier $\widehat{\varphi}_{n}$.

In place of estimating $\theta(K, \mu)$, Florens and Simar (2005) rather propose to estimate the pseudo-true value for the order- $m$ frontier:

$$
\theta_{m}(K, \mu)=\arg \min _{\theta \in \mathbb{R}^{k}} \int_{K}\left(\varphi_{m}(x)-\varphi_{\theta}(x)\right)^{2} d \mu(x),
$$

where $m \geq 1$ is an integer, by its nonparametric estimator:

$$
\widehat{\theta}_{m, n}(K, \mu)=\arg \min _{\theta \in \mathbb{R}^{k}} \int_{K}\left(\widehat{\varphi}_{m, n}(x)-\varphi_{\theta}(x)\right)^{2} d \mu(x)
$$


As an alternative, we rather propose in this paper to estimate a pseudo-true value for the order- $\alpha$ frontier, defined as

$$
\theta_{\alpha}(K, \mu)=\arg \min _{\theta \in \mathbb{R}^{k}} \int_{K}\left(q_{\alpha}(x)-\varphi_{\theta}(x)\right)^{2} d \mu(x) .
$$

Our quantile-based procedure has the merit to be more robust to extremes since the order- $\alpha$ quantile frontiers have the superiority of being more resistant to extremes than the FDH $\widehat{\varphi}_{n}$ and the order- $m$ frontiers $\widehat{\varphi}_{m, n}$ when estimating the true full frontier $\varphi$ as showed in Daouia and Ruiz-Gazen (2004). By a plug-in argument of the robust quantile frontier $\widehat{q}_{\alpha, n}$, we obtain the estimator

$$
\widehat{\theta}_{\alpha, n}(K, \mu)=\arg \min _{\theta \in \mathbb{R}^{k}} \int_{K}\left(\widehat{q}_{\alpha, n}(x)-\varphi_{\theta}(x)\right)^{2} d \mu(x) .
$$

As pointed in Florens and Simar (2005), the existence and uniqueness of the pseudo-true values (3.1), (3.3) and (3.5) are based on technical conditions (integrability, identification, structure of the functional space $\left\{\varphi_{\theta} \mid \theta \in \mathbb{R}^{k}\right\}$ ). We will not explicit these technical hypothesis here but the implicit set of functions we consider is the set of square integrable functions with respect to the density $d \mu(x)$. This set is an Hilbert space provided by its norm topology. If, for instance, the subset $\left\{\varphi_{\theta} \mid \theta \in \mathbb{R}^{k}\right\}$ is closed and convex, then the pseudo-true values exist and are unique. So, in what follows, we assume that these pseudo-true values exist and are unique.

We first derive the asymptotic distribution of $\widehat{\theta}_{\alpha, n}(K, \mu)$ as an estimator of $\theta_{\alpha}(K, \mu)$ and then we will propose robust and strongly consistent estimators of $\theta(K, \mu)$.

\section{Parametric approximation of the quantile frontier}

A natural choice for $\mu$ is the law $\mathbb{P}_{X}$ of $X$, but since $\mathbb{P}_{X}$ is usually unknown, we can define sample versions of the pseudo-true values by using the empirical probability measure $\widehat{\mathbb{P}}_{X, n}=$ $\frac{1}{n} \sum_{i=1}^{n} \delta_{X_{i}}$, putting a mass $1 / n$ at each $X_{i}$,

$$
\begin{aligned}
\theta_{\alpha}\left(K, \widehat{\mathbb{P}}_{X, n}\right) & =\arg \min _{\theta \in \mathbb{R}^{k}} \frac{1}{n} \sum_{i=1}^{n}\left(q_{\alpha}\left(X_{i}\right)-\varphi_{\theta}\left(X_{i}\right)\right)^{2} \mathbb{1}_{K}\left(X_{i}\right), \\
\widehat{\theta}_{\alpha, n}\left(K, \widehat{\mathbb{P}}_{X, n}\right) & =\arg \min _{\theta \in \mathbb{R}^{k}} \frac{1}{n} \sum_{i=1}^{n}\left(\widehat{q}_{\alpha, n}\left(X_{i}\right)-\varphi_{\theta}\left(X_{i}\right)\right)^{2} \mathbb{1}_{K}\left(X_{i}\right) .
\end{aligned}
$$

In order to simplify the presentation, we first restrict our parametric family to the class of linear models

$$
\varphi_{\theta}(x)=g^{T}(x) \theta
$$

where $g^{T}(x)=\left(g_{1}(x), \cdots, g_{k}(x)\right)$, with $g_{j}(\cdot)$ being known scalar functions. The next theorem establishes the asymptotic normality of our estimator. 
Theorem 3.1. If the scalar functions $g_{1}(\cdot), \cdots, g_{k}(\cdot)$ are bounded on $K$ and the conditions of Theorem 2.1 hold, then

$$
\sqrt{n}\left(\widehat{\theta}_{\alpha, n}\left(K, \widehat{\mathbb{P}}_{X, n}\right)-\theta_{\alpha}\left(K, \widehat{\mathbb{P}}_{X, n}\right)\right) \stackrel{\mathcal{L}}{\longrightarrow} N\left(0, M \Sigma^{\alpha} M\right) \quad \text { as } \quad n \rightarrow \infty
$$

where

$$
M=\left\{E\left[\mathbb{1}_{K}\left(X_{1}\right) g\left(X_{1}\right) g^{T}\left(X_{1}\right)\right]\right\}^{-1}, \quad \Sigma^{\alpha}=\operatorname{Var}_{F}\left\{E_{F}\left[H\left(\left(X_{2}, Y_{2}\right),\left(X_{1}, Y_{1}\right)\right) \mid\left(X_{1}, Y_{1}\right)\right]\right\}
$$

with $H(\cdot, \cdot)$ being defined in (A.13).

Let us now turn to the more general case where we consider a general parametric model and a general measure $\mu$. By applying Theorem 2.1 in conjunction with a technique of proof of Florens and Simar (2005), the next theorem shows the asymptotic normality of $\widehat{\theta}_{\alpha, n}(K, \mu)$.

Theorem 3.2. Assume that $\varphi$ and $\left\{\varphi_{\theta}, \theta \in \mathbb{R}^{k}\right\}$ are bounded on $K$ and that the conditions of Theorem 2.1 hold. Assume furthermore that $\theta \mapsto \varphi_{\theta}(x)$ is differentiable on $\mathbb{R}^{k}$ for any $x \in K$, that the differentiability at $\theta_{\alpha}(K, \mu)$ is uniform in $x \in K$ and that $A, A^{-1}, B$ and $\left.\int_{K} \frac{\partial \varphi_{\theta}}{\partial \theta}(x)\right|_{\theta=\theta_{\alpha}(K, \mu)} d \mu(x)$ exist, where

$$
\begin{aligned}
& A=\left.\left.\int_{K} \frac{\partial \varphi_{\theta}}{\partial \theta}(x)\right|_{\theta=\theta_{\alpha}(K, \mu)} \frac{\partial \varphi_{\theta}}{\partial \theta^{T}}(x)\right|_{\theta=\theta_{\alpha}(K, \mu)} d \mu(x) \\
& B=\left.\left.\int_{K} \int_{K} \frac{\partial \varphi_{\theta}}{\partial \theta}(x)\right|_{\theta=\theta_{\alpha}(K, \mu)} \frac{\partial \varphi_{\theta}}{\partial \theta^{T}}(z)\right|_{\theta=\theta_{\alpha}(K, \mu)} \Gamma_{\alpha}(x, z) d \mu(x) d \mu(z),
\end{aligned}
$$

with $\Gamma_{\alpha}(\cdot, \cdot)$ being described in (2.2). Then

$$
\sqrt{n}\left(\widehat{\theta}_{\alpha, n}(K, \mu)-\theta_{\alpha}(K, \mu)\right) \stackrel{\mathcal{L}}{\longrightarrow} N\left(0, A^{-1} B A^{-1}\right) .
$$

Note that for practical purposes (construction of confidence intervals,...), we could use bootstrap estimates of the variance matrix $A^{-1} B A^{-1}$, as suggested in details in Florens and Simar (2005, Appendix).

\section{Parametric approximation of the full frontier itself}

Florens and Simar (2005) prove that the estimator $\widehat{\theta}_{n}(K, \mu)$ defined in $(3.2)$, converges in probability to $\theta(K, \mu)$. First we will show below how this weak consistency can be extended to the almost sure sense. Putting $M(\theta)=\int_{K}\left(\varphi(x)-\varphi_{\theta}(x)\right)^{2} d \mu(x)$, it will be required that

$$
m_{\varepsilon}:=\inf \left\{M(\theta) \mid \theta \in \mathbb{R}^{k}: d(\theta, \theta(K, \mu)) \geq \varepsilon\right\}-M(\theta(K, \mu))>0
$$

for every $\varepsilon>0$, where $d(\cdot, \cdot)$ is the Euclidean metric on $\mathbb{R}^{k}$. Thus, $\theta(K, \mu)$ should be a well-separated point of minimum of the map $\theta \mapsto M(\theta)$. 
Theorem 3.3. Assume that $F_{X}, \varphi$ and $\varphi_{m}$ are continuous on a compact subset $K$ interior to the support of $X$, for every $m \geq 1$, and that the upper boundary $\nu>0$ of the support of $Y$ is finite. If furthermore $\delta:=\sup _{(x, \theta) \in K \times \mathbb{R}^{k}}\left|\varphi_{\theta}(x)\right|$ is finite and the condition (3.7) holds, then $\widehat{\theta}_{n}(K, \mu) \stackrel{\text { a.s. }}{\longrightarrow} \theta(K, \mu)$ as $n \rightarrow \infty$.

In particular, if the parametric model (1.1) is correctly specified for the frontier, then the estimator $\widehat{\theta}_{n}(K, \mu)$ converges almost surely to the value $\theta$ such that $\varphi=\varphi_{\theta}$.

On the other hand, Cazals et al. (2002) derived an estimator $\widehat{\varphi}_{m(n), n}$ of the full frontier function $\varphi$ by an appropriate choice of the order $m$ as a function of the sample size $n$. This estimator shares the same asymptotic distribution as the FDH estimator $\widehat{\varphi}_{n}$, but is more robust to extremes. Likewise, Aragon et al. (2005) suggested a quantile-type estimator $\widehat{q}_{\alpha(n), n}$ of $\varphi$, which keeps the same asymptotic distribution as $\widehat{\varphi}_{n}$ and $\widehat{\varphi}_{m(n), n}$, but is more stable and resistant to extremes. By applying the same technique of proof of Theorem 3.3 in conjunction with Theorem 2.1 and 2.3 in Daouia and Simar (2005), we obtain the almost sure convergence of $\widehat{\theta}_{m(n), n}(K, \mu)$ and $\widehat{\theta}_{\alpha(n), n}(K, \mu)$ when estimating $\theta(K, \mu)$ itself, provided ${ }^{3}$ that $m(n) \rightarrow \infty$, $m(n)(\log n / n)^{1 / 2} \rightarrow 0$ and $n(1-\alpha(n)) \rightarrow 0$ as $n \rightarrow \infty$. Even more strongly, the next theorem shows that $P\left[d\left(\widehat{\theta}_{m(n), n}(K, \mu), \theta(K, \mu)\right)>\varepsilon\right]$ and $P\left[d\left(\widehat{\theta}_{\alpha(n), n}(K, \mu), \theta(K, \mu)\right)>\varepsilon\right]$ converge to 0 at an exponential rate, for a fixed $\varepsilon>0$.

Theorem 3.4. Let the sequences $\{m(n) \geq 1\}$ and $\{0<\alpha(n)<1\}$ be such that

$$
\lim _{n \rightarrow \infty} m(n)=\infty, \quad \lim _{n \rightarrow \infty} m(n)(\log n / n)^{1 / 2}=0 \quad \text { and } \quad \lim _{n \rightarrow \infty} n(1-\alpha(n))=0 .
$$

Given the conditions of Theorem 3.3, there exists a constant $C \in(0, \infty)$ such that for all $\varepsilon>0$ and all $r>0, \lambda>1$ with $\lambda r<m_{\varepsilon} /(4(\nu+\delta) \mu(K))$, and for all $n$ large enough

$$
\begin{aligned}
P & {\left[d\left(\widehat{\theta}_{m(n), n}(K, \mu), \theta(K, \mu)\right)>\varepsilon\right] } \\
& \leq C\left\{\exp \left(-n r^{2}\left(\inf _{x \in K} F_{X}(x)\right)^{2} /(4 m(n) \nu)^{2}\right)+\exp \left(-n\left(1-\frac{1}{\lambda}\right)^{2}\left(\inf _{x \in K} F_{X}(x)\right)^{2}\right)\right\}
\end{aligned}
$$

and

$$
\begin{aligned}
P\left[d\left(\widehat{\theta}_{\alpha(n), n}(K, \mu), \theta(K, \mu)\right)>\varepsilon\right] \leq C\left\{\exp \left(-n r^{2}\left(\inf _{x \in K} F_{X}(x)\right)^{2} /(8 m(n) \nu)^{2}\right)\right. \\
\left.+\exp \left(-n\left(1-\frac{1}{\lambda}\right)^{2}\left(\inf _{x \in K} F_{X}(x)\right)^{2}\right)+\exp \left(-n(\lambda-1)^{2}\left(\sup _{x \in K} F_{X}(x)\right)^{2}\right)\right\} .
\end{aligned}
$$

\footnotetext{
${ }^{3}$ Note that when $\alpha(n) \rightarrow 1$ as $n \rightarrow \infty, \widehat{q}_{\alpha(n), n}(x)$ is a consistent estimator of $\varphi(x)$. As indicated in Theorem 2.3 in Daouia and Simar (2005), for $\widehat{q}_{\alpha(n), n}$ to converge uniformly with probability one to $\varphi, \alpha(n)$ should converge to one at the rate $n$. However, as pointed in Section 1, for $\widehat{q}_{\alpha(n), n}(x)$ to converge to the Weibull distribution, $\alpha(n)$ should converge faster to one, at the rate $n^{(p+2) /(p+1)}$.
} 
Note that the full frontier $\varphi(\cdot)$ is monotone and it is natural to try to incorporate this information into the estimation procedure. Daouia and Simar (2005) proposed nondecreasing estimators $\widehat{\varphi}_{m(n), n}^{\#}$ and $\widehat{q}_{\alpha(n), n}^{\#}$ of $\varphi$ by isotonizing the original estimators $\widehat{\varphi}_{m(n), n}$ and $\widehat{q}_{\alpha(n), n}$. These monotone estimators appear to be more stable and more robust to extreme values than the initial versions. Furthermore, they converge uniformly and almost surely to $\varphi$ under the same conditions of Theorem 3.3 (see Daouia and Simar, 2005, Theorems 2.1-2.3) and so, we can introduce the new estimators $\widehat{\theta}_{m(n), n}^{\#}(K, \mu)$ and $\widehat{\theta}_{\alpha(n), n}^{\#}(K, \mu)$ of $\theta(K, \mu)$ and easily establish their uniform almost sure convergence in the same way as the original ones $\widehat{\theta}_{m(n), n}(K, \mu)$ and $\widehat{\theta}_{\alpha(n), n}(K, \mu)$.

Note also that the analysis of the asymptotic distributions of $\widehat{\theta}_{n}(K, \mu), \widehat{\theta}_{m(n), n}(K, \mu)$, $\widehat{\theta}_{\alpha(n), n}(K, \mu)$ and their isotonized versions is more complexe since there is no functional convergence theorem for $\widehat{\varphi}_{n}, \widehat{\varphi}_{m(n), n}$ and $\widehat{q}_{\alpha(n), n}$ to $\varphi$. Only punctual convergence results to a Weibull distribution for $\widehat{\varphi}_{n}(x), \widehat{\varphi}_{m(n), n}(x)$ and $\widehat{q}_{\alpha(n), n}(x)$ are available, respectively, in Park et al. (2000), Cazals et al. (2002) and Aragon et al. (2005). We shall return to this topic in another paper.

All the results proved in our approach remain valid if we use $w(x) d x$ instead of $d \mu(x)$, where $w(x)$ is a given weight function which can be viewed as a density on $x$ weighting the error term.

\section{Numerical Illustrations}

In this section, we present three simulated examples as in Florens and Simar (2005) to illustrate our procedure, we also present a real data example on the activity of Air Controlers in Europe. We confirm the advantages of the robust semi-parametric estimators in the presence of outliers over their full frontier alternative (either using Shifted-OLS or the FDH filter as a first stage) and we compare in these examples the parametric quantile-type frontier approximations with the order- $m$ frontier approximations, indicating some advantages of the former over the latter.

\section{Example 1}

We first consider a case where the frontier function $\varphi$ is linear. We choose $(X, Y)$ uniformly distributed over the region $D=\{(x, y) \mid 0 \leq x \leq 1,0 \leq y \leq x\}$. Here $\varphi(x)=x, \varphi_{\theta}(x)=\theta x$ with $0 \leq \theta \leq 1$, and $F(y \mid x)=2 x^{-1} y-x^{-2} y^{2}$, for $0<x \leq 1$ and $0 \leq y \leq x$. The conditional $\alpha$-quantile is $q_{\alpha}(x)=x(1-\sqrt{1-\alpha})$, for $0<x \leq 1$ and $0 \leq \alpha \leq 1$, and the order- $m$ frontier can be computed as $\varphi_{m}(x)=x\left(1-A_{m}\right)$, where $A_{m}=\sum_{j=0}^{m}\left({ }_{j}{ }_{j}\right)(-1)^{m-j} 2^{j} /(2 m-j+1)$. Therefore $\theta_{\alpha}=1-\sqrt{1-\alpha}$ and $\theta_{m}=1-A_{m}$. 
The parametric approximations $\left\{\widehat{\theta}_{m, n}\right\}_{n}$ and $\left\{\widehat{\theta}_{\alpha, n}\right\}_{n}$ do not estimate in general the same quantity, except for the limiting case where $m$ tends to infinity and $\alpha$ to one. But in this particular example, if $\alpha=1-A_{m}^{2}$, then both $\theta_{\alpha}$ and $\theta_{m}$ coincide and so, the robust proposals of the two sequences of parametric approximations can be compared.

We illustrate in Figure 1 one sample of 100 observations generated according the above scenario, we chose $m=20$, so that $\alpha=0.9622$ for getting the same parametric approximations. We then compare the results when adding 3 outliers. As expected and already pointed in Florens and Simar (2005), in this scenario, the shifted-OLS behaves very badly. The interest here is focused on the comparison of the order- $m$ frontier with the $\alpha$-quantile, with their common FDH limit. When there are no outliers, the two fitted parametric frontier are, as expected by our choice of $m$ and $\alpha$, very similar but when the 3 outliers are added, the $\alpha$-quantile seems to resist more easily to the presence of the 3 serious outliers. We see also that the fit obtained by the FDH estimator is too sensitive to these outliers.

\section{Example 2}

We choose here a concave frontier given by the Cobb-Douglas model $Y=X^{1 / 2} \exp (-U)$, where $X$ is uniform on $[0,1]$ and $U$, independent of $X$, is Exponential with parameter $\lambda=3$. Here $\varphi(x)=x^{1 / 2}$ and $\varphi_{\theta}(x)=\theta x^{1 / 2}$ with $0 \leq \theta \leq 1$. The $\alpha$-quantile frontier is given by $q_{\alpha}(x)=x^{1 / 2} \theta_{\alpha}$, where $\theta_{\alpha}=\cos \left(\frac{\arccos (1-2 \alpha)+4 \pi}{3}\right)+\frac{1}{2}$, and the order- $m$ frontier can be computed as $\varphi_{m}(x)=x^{1 / 2} \theta_{m}$, where $\theta_{m}=1-B_{m}$ with $B_{m}=\sum_{j=0}^{m}\left(\begin{array}{c}m \\ j\end{array}\right)(-2)^{m-j} 3^{j} /(3 m-$ $j+1)$. In this particular case, if $\alpha=\frac{1}{2}\left(1-\cos \left[3 \arccos \left(\frac{1}{2}-B_{m}\right)-4 \pi\right]\right)$, then $\theta_{\alpha} \equiv \theta_{m}$ can be estimated by $\left\{\widehat{\theta}_{m, n}\right\}_{n}$ as well as $\left\{\widehat{\theta}_{\alpha, n}\right\}_{n}$.

We illustrate again, in Figure 2, one sample of 100 observations, where $m=20$ and $\alpha=$ 0.9612 for making the comparison possible. Then again, we compare the results when adding 3 outliers to the same sample. Here, the shifted-OLS behaves better since the inefficiency terms $U$ are independent of $X$. The comparison of the order- $m$ frontier with the $\alpha$-quantile leads to similar conclusion than in the preceding example: as expected, similar results when there are no outliers and again, the $\alpha$-quantile seems to resist more to the presence of the 3 outliers.

\section{Example 3}

We choose here, as in Florens and Simar (2005) the same scenario as in the preceding example but we introduce heterogeneity in the inefficiency term ( $U$ is not independent of $X$ ): here we choose $E(U \mid X)=\frac{2}{3}(1-X)$ so that $E(U)=1 / 3$ as above in Example 2. We do not have explicit analytical expressions for the $\varphi_{m}(x)$ nor for $q_{\alpha}(x)$. But, as suggested by Aragon et 
al. (2005), for ease of comparison, we fixed a value $\alpha=0.95$ and then we selected a value of $m$ in the simulated sample that provided approximatively the same percentage of observed points outside the two estimated partial frontiers (i.e. with values of $\left\{\widehat{\theta}_{m, n}\right\}$ and of $\left\{\widehat{\theta}_{\alpha, n}\right\}$ greater than one). In our example below, this provided a value of $m=38$.

Figure 3 displays the results (again, one sample of 100 observations without and with 3 added outliers). Here, the shifted-OLS is a catastrophe (as pointed in Florens and Simar, 2005). The comparison of the order- $m$ frontier with the $\alpha$-quantile leads again to similar conclusion than in the two preceding examples: similar results when there are no outliers and better robustness to the three outliers for the $\alpha$-quantile fitted frontier.

\section{A real data set}

We could use our approach to any model with multiple inputs but in order to provide pictures in two dimensions, we illustrate our estimators with a real data coming from the efficiency analysis of Air Controllers in Europe (Mouchart and Simar, 2002). We have data on activity of 37 European air controllers in the year 2000. The activity of each controller can be described by one input (an aggregate factor of different kind of labor) and one output (an aggregate factor of the activity produced, based on the number of air movements controlled, the number flight hours controlled, ...).

Here we have $n=37$ observations and as explained in the preceding example, we selected $\alpha=0.95$ and then fixed the value of $m$, for comparison, leaving the same percentage of points outside the two partial frontiers: this gave us $m=41$ with $13,51 \%$ of the observations above the two estimated partial frontiers. The computation time is so fast (less than 0.15 seconds for producing the estimates), that a grid of values for the tuning parameters ( $m$ and $\alpha$ ) can be chosen and then the practitioner could proceed to a sensitivity analysis. We also chose a Cobb-Douglas specification for the frontier model. Figure 4 displays the obtained results.

Clearly, the shifted-OLS seems to be outperformed by the other estimators, since it is based on a restrictive hypothesis of homogeneous distribution of the inefficiencies. The fit obtained through the first step FDH filter provide a sensible estimate but is very sensitive to extreme values (one around $x=2.8$ and the other on the right around $x=7.5$ ). Again, the fit based on the order- $m$ filter is slightly more resistant to these extreme points but since $m$ is large, the fit is not far from the FDH fit. As expected, the fit obtained with the $\alpha$-quantile frontier is much more robust to the extremes.

Just for illustrative purpose, we reproduce in Table 1 the point estimates of the fitted Cobb-Douglas production frontier. As explained in Florens and Simar (2005), standard deviations of the parameters obtained through the order- $m$ and the $\alpha$-quantile frontier can be obtained by simple bootstrap. The other two approaches (full-frontier estimates) cannot 
provide, up to now, estimates of the standard deviations of the estimates.

\begin{tabular}{|l||c|c|}
\hline method & Intercept & Elasticity \\
\hline Shifted-OLS & 0.8238 & 0.8833 \\
FDH & 0.5886 & 0.8838 \\
$\alpha$-quantile & 0.5554 & 0.7798 \\
order- $m$ & 0.5796 & 0.8553 \\
\hline
\end{tabular}

Table 1: Point estimates of the Cobb-Douglas parameters following the different approaches. Here $\alpha=0.95$ and $m=41$.

\section{Conclusion}

In this paper we have shown how the parametric approximation of $\alpha$-quantile frontiers offers an attractive alternative to the approximation of the order- $m$ frontier, as proposed by Florens and Simar (2005). The former benefits from better robustness properties to outliers or extreme values than the latter, both approaches being more robust than the parametric approximation of the full frontier using the FDH as first step.

It confirms also that these approaches are much more appealing than the classical parametric estimators of the frontier, that are mostly based on regression ideas and fit the shape of the center of the cloud of points rather than its boundary.

The paper provides some asymptotic theory. We first prove a functional central limit theorem for the quantile-type frontier process. Then we establish the asymptotic normality of the resulting estimators of the parametric approximation of the partial order- $\alpha$ frontier.

When estimating the parameters of the full frontier itself by using the FDH as first step, we improve the weak consistency obtained by Florens and Simar (2005). When the robust order- $m$ or order- $\alpha$ partial frontiers are used in the first step, by chosing $m$ and $\alpha$ as appropriate functions of $n$, we show that the obtained estimators converge exponentially fast. Finally the good behavior of our method in finite samples is illustrated through simulated and real data sets.

\section{Appendix: Lemmas and Proofs.}

\section{A.1 Proof of Theorem 2.1}

Let $\nu>0$ be the upper boundary of the support of $Y$. The domain $\mathbb{D} \subset \mathbb{R}^{p+1}$ is used to denote any fixed compact subset which contains both $K \times[-\nu, \nu]$ and the support $\Psi$ of 
$(X, Y)$. Define the domain $\mathbb{G}$ of distribution functions $G(\cdot, \cdot)$ on $\mathbb{R}_{+}^{p} \times \mathbb{R}_{+}$whose supports are also contained in $\mathbb{D}$ and such that

$$
G(x, \infty)>0 \forall x \in K, \quad \inf \{y \geq 0 \mid G(\infty, y)=1\} \leq \nu
$$

For any $G \in \mathbb{G}$ and any $x \in K$, let $G^{-1}(\alpha \mid x):=\inf \{y \geq 0 \mid G(y \mid x) \geq \alpha\}$ denotes the $\alpha$ th quantile of the conditional distribution function $G(\cdot \mid x)=G(x, \cdot) / G(x, \infty) \equiv G(x, \cdot) / G(x, \nu)$. Then, for any $G \in \mathbb{G}$ and any $x \in K$, we have

$$
0 \leq G^{-1}(\alpha \mid x) \leq G^{-1}(1 \mid x) \leq \inf \{y \geq 0 \mid G(\infty, y)=1\} \leq \nu
$$

Hence the conditional quantile transformation $G^{-1}(\alpha \mid \cdot): x \mapsto G^{-1}(\alpha \mid x)$ as a map $K \rightarrow[0, \nu]$ is bounded on $K$ and so, we write $G^{-1}(\alpha \mid \cdot) \in L^{\infty}(K)$. Now consider $\phi: G \mapsto G^{-1}(\alpha \mid \cdot)$ as a map $\mathbb{G} \subset L^{\infty}(\mathbb{D}) \longrightarrow L^{\infty}(K)$. We obtain the following result.

Lemma A.1. Under the conditions of Theorem 2.1, the functional $\phi$ is Hadamard-differentiable at $F \in \mathbb{G}$ tangentially to the set $C(\mathbb{D})$ of continuous functions on $\mathbb{D}$. The derivative is the map $\phi_{F}^{\prime}: h \mapsto \phi_{F}^{\prime}(h)$ as a map $C(\mathbb{D}) \longrightarrow L^{\infty}(K)$, where

$$
\phi_{F}^{\prime}(h): x \mapsto \frac{\alpha h(x, \nu)-h\left(x, q_{\alpha}(x)\right)}{f\left(q_{\alpha}(x) \mid x\right) F_{X}(x)} .
$$

Proof. Let $h_{t} \rightarrow h$ uniformly in $L^{\infty}(\mathbb{D})$, where $h \in C(\mathbb{D})$ and $F+t h_{t} \in \mathbb{G}$ for all small $t>0$. Write $q_{\alpha t}$ for $\phi\left(F+t h_{t}\right)$. Following the definition of the Hadamard differentiability (see van der Vaart (1998), p.296), we shall show that $\left(q_{\alpha t}(x)-q_{\alpha}(x)\right) / t$ converges to $(\alpha h(x, \nu)-$ $\left.h\left(x, q_{\alpha}(x)\right)\right) / f\left(q_{\alpha}(x) \mid x\right) F_{X}(x)$ as $t \searrow 0$, uniformly in $x \in K$. By the definition of $\phi$, we have for every $x \in K$ and every $\varepsilon>0$,

$$
\left(F+t h_{t}\right)\left(q_{\alpha t}(x)-\varepsilon \mid x\right)<\alpha \leq\left(F+t h_{t}\right)\left(q_{\alpha t}(x) \mid x\right) .
$$

We choose $\varepsilon=\varepsilon_{t}=o(t)$ as $t \searrow 0$. Because $G(x, \infty)=G(x, \nu)>0$ for any $G \in \mathbb{G}$ and any $x \in K$, we have $\left(F+t h_{t}\right)\left(x, q_{\alpha t}(x)-\varepsilon_{t}\right)<\alpha\left(F+t h_{t}\right)(x, \nu) \leq\left(F+t h_{t}\right)\left(x, q_{\alpha t}(x)\right)$. Then

$$
\begin{gathered}
F\left(q_{\alpha t}(x)-\varepsilon_{t} \mid x\right)+\frac{t h_{t}\left(x, q_{\alpha t}(x)-\varepsilon_{t}\right)-\alpha t h_{t}(x, \nu)}{F_{X}(x)}<\alpha \\
\leq F\left(q_{\alpha t}(x) \mid x\right)+\frac{t h_{t}\left(x, q_{\alpha t}(x)\right)-\alpha t h_{t}(x, \nu)}{F_{X}(x)}
\end{gathered}
$$

Since $\varepsilon_{t} \searrow 0$ as $t \searrow 0, \varepsilon_{t}<\nu$ for all $t$ small enough, and so $q_{\alpha t}(x)-\varepsilon_{t} \in[-\nu, \nu]$ for every $x \in K$ and all $t$ sufficiently small. Therefore $\sup _{x \in K}\left|h_{t}\left(x, q_{\alpha t}(x)-\varepsilon_{t}\right)-h\left(x, q_{\alpha t}(x)-\varepsilon_{t}\right)\right| \leq$ $\sup _{(x, y) \in K \times[-\nu, \nu]}\left|h_{t}(x, y)-h(x, y)\right|$, for all small $t$. Since $h_{t}$ converges uniformly to $h$ on $K \times[-\nu, \nu] \subset \mathbb{D}$, we obtain $t h_{t}\left(x, q_{\alpha t}(x)-\varepsilon_{t}\right)=\operatorname{th}\left(x, q_{\alpha t}(x)-\varepsilon_{t}\right)+o(t)$ uniformly in $x \in K$. 
Likewise, $t h_{t}\left(x, q_{\alpha t}(x)\right)=t h\left(x, q_{\alpha t}(x)\right)+o(t)$ and $-\alpha t h_{t}(x, \nu)=-\alpha t h(x, \nu)-\alpha o(t)$, where the $o(t)$ terms are uniform in $x \in K$. By using the fact that $\sup _{(x, y) \in K \times \mathbb{R}} f(y \mid x)<\infty$, we also have $F\left(q_{\alpha t}(x)-\varepsilon_{t} \mid x\right)=F\left(q_{\alpha t}(x) \mid x\right)+O\left(\varepsilon_{t}\right)$, uniformly in $x \in K$. Hence

$$
\begin{aligned}
F\left(q_{\alpha t}(x) \mid x\right)+O\left(\varepsilon_{t}\right)+ & \frac{t h\left(x, q_{\alpha t}(x)-\varepsilon_{t}\right)+o(t)-\alpha \operatorname{th}(x, \nu)-\alpha o(t)}{F_{X}(x)}<\alpha \\
& \leq F\left(q_{\alpha t}(x) \mid x\right)+\frac{t h\left(x, q_{\alpha t}(x)\right)+o(t)-\alpha \operatorname{th}(x, \nu)-\alpha o(t)}{F_{X}(x)} .
\end{aligned}
$$

By replacing $\alpha$ in the middle of inequalities by $F\left(q_{\alpha}(x) \mid x\right)$, we get

$$
\begin{gathered}
-\frac{\operatorname{th}\left(x, q_{\alpha t}(x)\right)+o(t)-\alpha \operatorname{th}(x, \nu)-\alpha o(t)}{F_{X}(x)} \leq F\left(q_{\alpha t}(x) \mid x\right)-F\left(q_{\alpha}(x) \mid x\right) \\
<-O\left(\varepsilon_{t}\right)-\frac{\operatorname{th}\left(x, q_{\alpha t}(x)-\varepsilon_{t}\right)+o(t)-\alpha \operatorname{th}(x, \nu)-\alpha o(t)}{F_{X}(x)} .
\end{gathered}
$$

Let us show that $q_{\alpha t}(x) \rightarrow q_{\alpha}(x)$ uniformly in $x \in K$, as $t \searrow 0$. In view of the uniform differentiability, in $x \in K$, of $F(\cdot \mid x)$ at $q_{\alpha}(x)$, there exists a $\xi_{0}>0$ such that for all $|\xi|<\xi_{0}$ we have $\sup _{x \in K}\left|\frac{F\left(q_{\alpha}(x)+\xi \mid x\right)-\alpha}{\xi}-f\left(q_{\alpha}(x) \mid x\right)\right|<\inf _{x \in K} f\left(q_{\alpha}(x) \mid x\right) / 2$. Let $0<\delta<\xi_{0}$. Then $\inf _{x \in K} f\left(q_{\alpha}(x) \mid x\right) / 2<\frac{F\left(q_{\alpha}(x)+\delta \mid x\right)-\alpha}{\delta}$ and $\inf _{x \in K} f\left(q_{\alpha}(x) \mid x\right) / 2<\frac{\alpha-F\left(q_{\alpha}(x)-\delta \mid x\right)}{\delta}$, for all $x \in K$. Whence $\sup _{x \in K} F\left(q_{\alpha}(x)-\delta \mid x\right)<\alpha<\inf _{x \in K} F\left(q_{\alpha}(x)+\delta \mid x\right)$. Let $\Delta_{\delta}=\left[\inf _{x \in K} F\left(q_{\alpha}(x)+\right.\right.$ $\delta \mid x)-\alpha] \wedge\left[\alpha-\sup _{x \in K} F\left(q_{\alpha}(x)-\delta \mid x\right)\right]$. We have

$$
\begin{aligned}
\sup _{x \in K} \frac{\left|h_{t}\left(x, q_{\alpha t}(x)\right)-\alpha h_{t}(x, \nu)\right|}{F_{X}(x)} \leq & {\left[\sup _{x \in K}\left|h_{t}\left(x, q_{\alpha t}(x)\right)-h\left(x, q_{\alpha t}(x)\right)\right|+\sup _{x \in K}\left|h\left(x, q_{\alpha t}(x)\right)\right|\right.} \\
& \left.+\sup _{x \in K}\left|h_{t}(x, \nu)-h(x, \nu)\right|+\sup _{x \in K}|h(x, \nu)|\right] / \inf _{x \in K} F_{X}(x) .
\end{aligned}
$$

The fact that $q_{\alpha t}(x) \in[0, \nu]$, for every $x \in K$, implies that $\sup _{x \in K}\left|h\left(x, q_{\alpha t}(x)\right)\right|$ is majored by $\sup _{(x, y) \in K \times[0, \nu]}|h(x, y)|$ which is finite since $h$ is bounded on $K \times[-\nu, \nu] \subset \mathbb{D}$. Likewise $\sup _{x \in K}|h(x, \nu)|$ is finite. Hence, the uniform convergence of $h_{t}$ to $h$ yields

$$
\sup _{x \in K} \frac{\left|t h_{t}\left(x, q_{\alpha t}(x)\right)-\alpha t h_{t}(x, \nu)\right|}{F_{X}(x)} \longrightarrow 0 \quad \text { as } \quad t \searrow 0 .
$$

Since $q_{\alpha t}(x)-\varepsilon_{t} \in[-\nu, \nu]$ for every $x \in K$ and all $\operatorname{small}_{t}, \sup _{x \in K}\left|h\left(x, q_{\alpha t}(x)-\varepsilon_{t}\right)\right|$ is majored by $\sup _{(x, y) \in K \times[-\nu, \nu]}|h(x, y)|$ which is finite since $h \in L^{\infty}(\mathbb{D})$. Thus, we obtain in the same way as for (A.4) that

$$
\sup _{x \in K} \frac{\left|t h_{t}\left(x, q_{\alpha t}(x)-\varepsilon_{t}\right)-\alpha t h_{t}(x, \nu)\right|}{F_{X}(x)} \longrightarrow 0 \quad \text { as } \quad t \searrow 0 .
$$


Regarding (A.4) and (A.5), there exists $t_{\delta}>0$ such that for all $t<t_{\delta}$ and for every $x \in K$,

$$
\begin{aligned}
& F\left(q_{\alpha}(x)-\delta \mid x\right) \leq \alpha-\Delta_{\delta}< \\
& \alpha-\frac{t h_{t}\left(x, q_{\alpha t}(x)\right)-\alpha t h_{t}(x, \nu)}{F_{X}(x)} \text { and } \quad \alpha-\frac{t h_{t}\left(x, q_{\alpha t}(x)-\varepsilon_{t}\right)-\alpha t h_{t}(x, \nu)}{F_{X}(x)} \\
& <\alpha+\Delta_{\delta} \leq F\left(q_{\alpha}(x)+\delta \mid x\right) .
\end{aligned}
$$

By using the inequalities (A.2), we get $F\left(q_{\alpha}(x)-\delta \mid x\right)<F\left(q_{\alpha t}(x) \mid x\right)$ and $F\left(q_{\alpha t}(x)-\varepsilon_{t} \mid x\right)<$ $F\left(q_{\alpha}(x)+\delta \mid x\right)$. It follows by the monotonicity of $F(\cdot \mid x)$ that $-\delta<q_{\alpha t}(x)-q_{\alpha}(x)<\delta+\varepsilon_{t}$ uniformly in $x \in K$, and thus we conclude that

$$
\sup _{x \in K}\left|q_{\alpha t}(x)-q_{\alpha}(x)\right| \longrightarrow 0 \quad \text { as } \quad t \searrow 0 .
$$

It can be then easily seen from the uniform differentiability of $F(\cdot \mid x)$ at $q_{\alpha}(x)$ that

$$
\sup _{x \in K}\left|\frac{F\left(q_{\alpha t}(x) \mid x\right)-F\left(q_{\alpha}(x) \mid x\right)}{q_{\alpha t}(x)-q_{\alpha}(x)}-f\left(q_{\alpha}(x) \mid x\right)\right| \longrightarrow 0 \quad \text { as } \quad t \searrow 0 .
$$

This yields $F\left(q_{\alpha t}(x) \mid x\right)-F\left(q_{\alpha}(x) \mid x\right)=\left(q_{\alpha t}(x)-q_{\alpha}(x)\right) f\left(q_{\alpha}(x) \mid x\right)+\left(q_{\alpha t}(x)-q_{\alpha}(x)\right) o(1)$, uniformly in $x \in K$. Therefore

$$
\begin{aligned}
- & \frac{\left(q_{\alpha t}(x)-q_{\alpha}(x)\right)}{t f\left(q_{\alpha}(x) \mid x\right)} o(1)-\frac{\operatorname{th}\left(x, q_{\alpha t}(x)\right)+o(t)-\alpha \operatorname{th}(x, \nu)-\alpha o(t)}{t f\left(q_{\alpha}(x) \mid x\right) F_{X}(x)} \leq \frac{\left(q_{\alpha t}(x)-q_{\alpha}(x)\right)}{t} \\
& <-\frac{\left(q_{\alpha t}(x)-q_{\alpha}(x)\right)}{t f\left(q_{\alpha}(x) \mid x\right)} o(1)-\frac{O\left(\varepsilon_{t}\right)}{t f\left(q_{\alpha}(x) \mid x\right)}-\frac{\operatorname{th}\left(x, q_{\alpha t}(x)-\varepsilon_{t}\right)+o(t)-\alpha \operatorname{th}(x, \nu)-\alpha o(t)}{t f\left(q_{\alpha}(x) \mid x\right) F_{X}(x)} .
\end{aligned}
$$

Thus we arrive at

$$
\begin{gathered}
\sup _{x \in K}\left|\frac{q_{\alpha t}(x)-q_{\alpha}(x)}{t}-\frac{\alpha h(x, \nu)-h\left(x, q_{\alpha}(x)\right)}{f\left(q_{\alpha}(x) \mid x\right) F_{X}(x)}\right| \leq \frac{1}{\inf _{x \in K} f\left(q_{\alpha}(x) \mid x\right) F_{X}(x)} \\
\times\left(\sup _{x \in K}\left|h\left(x, q_{\alpha t}(x)-\varepsilon_{t}\right)-h\left(x, q_{\alpha}(x)\right)\right|+\sup _{x \in K}\left|h\left(x, q_{\alpha t}(x)\right)-h\left(x, q_{\alpha}(x)\right)\right|+\frac{o(t)}{t}\right) \\
\quad+\frac{1}{\inf _{x \in K} f\left(q_{\alpha}(x) \mid x\right)}\left(\sup _{x \in K} \frac{\left|q_{\alpha t}(x)-q_{\alpha}(x)\right|}{t} o(1)+\frac{O\left(\varepsilon_{t}\right)}{t}\right) .
\end{gathered}
$$

Because $h$ is uniformly continuous on the compact $\mathbb{D}$ and $\varepsilon_{t} \rightarrow 0$, it follows from (A.6) that

$$
\sup _{x \in K}\left|h\left(x, q_{\alpha t}(x)\right)-h\left(x, q_{\alpha}(x)\right)\right| \rightarrow 0, \quad \sup _{x \in K}\left|h\left(x, q_{\alpha t}(x)-\varepsilon_{t}\right)-h\left(x, q_{\alpha}(x)\right)\right| \rightarrow 0
$$

as $t \searrow 0$. Now, let us show that $\sup _{x \in K}\left|q_{\alpha t}(x)-q_{\alpha}(x)\right| / t$ is bounded as $t \searrow 0$. It can be easily seen from (A.3) that

$$
\begin{aligned}
\inf _{x \in K}\left|\frac{F\left(q_{\alpha t}(x) \mid x\right)-F\left(q_{\alpha}(x) \mid x\right)}{q_{\alpha t}(x)-q_{\alpha}(x)}\right| & \times \sup _{x \in K} \frac{\left|q_{\alpha t}(x)-q_{\alpha}(x)\right|}{t} \\
\leq \frac{O\left(\varepsilon_{t}\right)}{t}+\frac{1}{\inf _{x \in K} F_{X}(x)} & {\left[\sup _{x \in K}\left|h\left(x, q_{\alpha t}(x)\right)-h\left(x, q_{\alpha}(x)\right)\right|+\frac{o(t)}{t}+\sup _{x \in K}|h(x, \nu)|\right.} \\
& \left.+\sup _{x \in K}\left|h\left(x, q_{\alpha}(x)\right)\right|+\sup _{x \in K}\left|h\left(x, q_{\alpha t}(x)-\varepsilon_{t}\right)-h\left(x, q_{\alpha}(x)\right)\right|\right] .
\end{aligned}
$$


Since $q_{\alpha}(x) \in[0, \nu]$ for every $x \in K, \sup _{x \in K}\left|h\left(x, q_{\alpha}(x)\right)\right|$ is majored by $\sup _{(x, y) \in \mathbb{D}}|h(x, y)|$ which is finite. Likewise $\sup _{x \in K}|h(x, \nu)|$ is finite. Furthermore, we have by (A.7),

$$
\inf _{x \in K}\left|\frac{F\left(q_{\alpha t}(x) \mid x\right)-F\left(q_{\alpha}(x) \mid x\right)}{q_{\alpha t}(x)-q_{\alpha}(x)}\right| \longrightarrow \inf _{x \in K} f\left(q_{\alpha}(x) \mid x\right)>0 \quad \text { as } \quad t \searrow 0 .
$$

Hence, by using these results in conjunction with (A.9) and (A.10), we get

$$
q_{\alpha t}(x)-q_{\alpha}(x)=O(t), \text { uniformly in } x \in K \text {. }
$$

Finally, by combining (A.8), (A.9) and (A.11), we obtain the desired uniform convergence in $x \in K$ of $\left(q_{\alpha t}(x)-q_{\alpha}(x)\right) / t$ to $\left(\alpha h(x, \nu)-h\left(x, q_{\alpha}(x)\right)\right) / f\left(q_{\alpha}(x) \mid x\right) F_{X}(x)$, as $t \searrow 0$.

On the other hand, the empirical process $\sqrt{n}\left(\widehat{F}_{n}-F\right)$ converges in distribution in $L^{\infty}\left(\overline{\mathbb{R}}^{p+1}\right)$ to $\mathbb{F}$, a $p+1$ dimensional F-Brownian bridge (see , e.g., van der Vaart and Wellner, 1996, p. 82). $\mathbb{F}$ is a Gaussian process with zero mean and covariance function $E\left(\mathbb{F}\left(t_{1}\right) \mathbb{F}\left(t_{2}\right)\right)=F\left(t_{1} \wedge t_{2}\right)-F\left(t_{1}\right) F\left(t_{2}\right)$, for all $t_{1}, t_{2} \in \overline{\mathbb{R}}^{p+1}$.

Since $L^{\infty}\left(\overline{\mathbb{R}}^{p+1}\right) \subset L^{\infty}(\mathbb{D})$, we obtain in view of Lemma 18.13 of van der Vaart (1998, p.261) that $\sqrt{n}\left(\widehat{F}_{n}-F\right)$ also converges in distribution to $\mathbb{F}$ in $L^{\infty}(\mathbb{D})$. Because $F \in C(\mathbb{D})$, the sample paths of the $F$-Brownian bridge are continuous on $\mathbb{D}$.

By Lemma A.1, the map $\phi: \mathbb{G} \subset L^{\infty}(\mathbb{D}) \longrightarrow L^{\infty}(K)$ is Hadamard-differentiable at $F$ tangentially to $C(\mathbb{D})$. Thus, the functional delta method (see Theorem 20.8 of van der Vaart, 1998, p.297) can be applied. It implies that $\sqrt{n}\left(\phi\left(\widehat{F}_{n}\right)-\phi(F)\right)=\sqrt{n}\left(\widehat{q}_{\alpha, n}-q_{\alpha}\right)$ converges in distribution to $\phi_{F}^{\prime}(\mathbb{F})$ in $L^{\infty}(K)$. Since the process $\mathbb{F}$ is Gaussian and the operator $\phi_{F}^{\prime}$ is linear, the limiting process $\phi_{F}^{\prime}(\mathbb{F})$ is Gaussian with zero mean and covariance function given, for any $x^{1}, x^{2} \in K$, by

$$
\Gamma_{\alpha}\left(x^{1}, x^{2}\right)=E\left[\frac{\alpha \mathbb{F}\left(x^{1}, \nu\right)-\mathbb{F}\left(x^{1}, q_{\alpha}\left(x^{1}\right)\right)}{f\left(q_{\alpha}\left(x^{1}\right) \mid x^{1}\right) F_{X}\left(x^{1}\right)} \times \frac{\alpha \mathbb{F}\left(x^{2}, \nu\right)-\mathbb{F}\left(x^{2}, q_{\alpha}\left(x^{2}\right)\right)}{f\left(q_{\alpha}\left(x^{2}\right) \mid x^{2}\right) F_{X}\left(x^{2}\right)}\right] .
$$

\section{A.2 Proof of Corollary 2.1}

Under the conditions of Lemma A.1, the remainder term $R_{n}^{\alpha}(x)$ of $(2.1)$, considered as a process indexed by $x \in K$, can be expressed in the following way

$$
\sqrt{n} R_{n}^{\alpha}=\sqrt{n}\left(\widehat{q}_{\alpha, n}-q_{\alpha}\right)-\phi_{F}^{\prime}\left(\sqrt{n}\left(\widehat{F}_{n}-F\right)\right)
$$

where $\phi_{F}^{\prime}(h)$ is described in (A.1) for any $h \in L^{\infty}\left(\overline{\mathbb{R}}^{p+1}\right)$. Because the linear operator $\phi_{F}^{\prime}$ is continuous from $L^{\infty}\left(\overline{\mathbb{R}}^{p+1}\right)$ into $L^{\infty}(K)$, it follows from the continuous mapping theorem that the process $\phi_{F}^{\prime}\left(\sqrt{n}\left(\widehat{F}_{n}-F\right)\right)$, indexed by $x \in K$, converges in distribution to the tight Gaussian process $\phi_{F}^{\prime}(\mathbb{F})$ in $L^{\infty}(K)$. Therefore, according to Theorem 1.5.4 of van der Vaart 
and Wellner (1996, p.35), both processes $\phi_{F}^{\prime}\left(\sqrt{n}\left(\widehat{F}_{n}-F\right)\right)$ and $\sqrt{n}\left(\widehat{q}_{\alpha, n}-q_{\alpha}\right)$ are asymptotically tight under the conditions of Theorem 2.1. The difference process $\sqrt{n} R_{n}^{\alpha}$, given by (A.12), is then asymptotically tight under the same conditions (see, e.g., Theorem 1.5.6 of van der Vaart and Wellner, p.36). Moreover, its marginals $\left(\sqrt{n} R_{n}^{\alpha}\left(x^{1}\right), \cdots, \sqrt{n} R_{n}^{\alpha}\left(x^{r}\right)\right)$ converge in probability to $0 \in \mathbb{R}^{r}$, for every $x^{1}, \cdots, x^{r} \in K$. Thus the nuisance process $\sqrt{n} R_{n}^{\alpha}$, indexed by $x \in K$, converges in distribution to the process zero in $L^{\infty}(K)$ in view of the second part of Theorem 1.5.4 of van der Vaart and Wellner (1996). Since the map $g \mapsto \sup _{x \in K}|g(x)|$ from $L^{\infty}(K)$ into $\mathbb{R}$ is continuous with respect to the supremum norm, the continuous-mapping theorem immediately implies the result.

\section{A.3 Proof of Theorem 3.1}

The values $\widehat{\theta}_{\alpha, n}\left(K, \widehat{\mathbb{P}}_{X, n}\right)$ and $\theta_{\alpha}\left(K, \widehat{\mathbb{P}}_{X, n}\right)$ have the following explicit expressions

$$
\begin{aligned}
\widehat{\theta}_{\alpha, n}\left(K, \widehat{\mathbb{P}}_{X, n}\right) & =\left\{\frac{1}{n} \sum_{i=1}^{n} \mathbb{1}_{K}\left(X_{i}\right) g\left(X_{i}\right) g^{T}\left(X_{i}\right)\right\}^{-1}\left[\frac{1}{n} \sum_{i=1}^{n} \mathbb{1}_{K}\left(X_{i}\right) \widehat{q}_{\alpha, n}\left(X_{i}\right) g\left(X_{i}\right)\right], \\
\theta_{\alpha}\left(K, \widehat{\mathbb{P}}_{X, n}\right) & =\left\{\frac{1}{n} \sum_{i=1}^{n} \mathbb{1}_{K}\left(X_{i}\right) g\left(X_{i}\right) g^{T}\left(X_{i}\right)\right\}^{-1}\left[\frac{1}{n} \sum_{i=1}^{n} \mathbb{1}_{K}\left(X_{i}\right) q_{\alpha}\left(X_{i}\right) g\left(X_{i}\right)\right] .
\end{aligned}
$$

The estimation error is then given by

$$
\widehat{\theta}_{\alpha, n}\left(K, \widehat{\mathbb{P}}_{X, n}\right)-\theta_{\alpha}\left(K, \widehat{\mathbb{P}}_{X, n}\right)=\left\{\frac{1}{n} \sum_{i=1}^{n} \mathbb{1}_{K}\left(X_{i}\right) g\left(X_{i}\right) g^{T}\left(X_{i}\right)\right\}^{-1} V_{n}
$$

where $V_{n}:=\frac{1}{n} \sum_{i=1}^{n} \mathbb{1}_{K}\left(X_{i}\right) g\left(X_{i}\right)\left(\widehat{q}_{\alpha, n}\left(X_{i}\right)-q_{\alpha}\left(X_{i}\right)\right)$. We have from (2.1), for any $x \in K$,

$$
\begin{aligned}
\widehat{q}_{\alpha, n}(x)-q_{\alpha}(x)= & \frac{1}{n} \sum_{j=1}^{n}\left(1 / f\left(q_{\alpha}(x) \mid x\right) F_{X}(x)\right) \\
& \times\left\{\alpha \mathbb{I}\left(X_{j} \leq x\right)-\mathbb{I}\left(X_{j} \leq x, Y_{j} \leq q_{\alpha}(x)\right)\right\}+R_{n}^{\alpha}(x) .
\end{aligned}
$$

Then

$$
V_{n}=\frac{1}{n^{2}} \sum_{i=1}^{n} \sum_{j=1}^{n} H\left(\left(X_{i}, Y_{i}\right),\left(X_{j}, Y_{j}\right)\right)+\frac{1}{n} \sum_{i=1}^{n} \mathbb{1}_{K}\left(X_{i}\right) g\left(X_{i}\right) R_{n}^{\alpha}\left(X_{i}\right)
$$

where

$$
\begin{aligned}
H\left(\left(X_{i}, Y_{i}\right),\left(X_{j}, Y_{j}\right)\right)= & \mathbb{1}_{K}\left(X_{i}\right) g\left(X_{i}\right)\left(1 / f\left(q_{\alpha}\left(X_{i}\right) \mid X_{i}\right) F_{X}\left(X_{i}\right)\right) \\
& \times\left\{\alpha \mathbb{I}\left(X_{j} \leq X_{i}\right)-\mathbb{I}\left(X_{j} \leq X_{i}, Y_{j} \leq q_{\alpha}\left(X_{i}\right)\right)\right\} .
\end{aligned}
$$

Since $\sum_{i=1}^{n} \sum_{j=1}^{n} H\left(\left(X_{i}, Y_{i}\right),\left(X_{j}, Y_{j}\right)\right)=\sum_{i=1}^{n} \sum_{j=1}^{n} H\left(\left(X_{j}, Y_{j}\right),\left(X_{i}, Y_{i}\right)\right)$, we have

$$
\frac{1}{n^{2}} \sum_{i=1}^{n} \sum_{j=1}^{n} H\left(\left(X_{i}, Y_{i}\right),\left(X_{j}, Y_{j}\right)\right)=\frac{1}{n^{2}} \sum_{i=1}^{n} \sum_{j=1}^{n} \mathcal{H}\left(\left(X_{i}, Y_{i}\right),\left(X_{j}, Y_{j}\right)\right)
$$


where $\mathcal{H}\left(\left(X_{i}, Y_{i}\right),\left(X_{j}, Y_{j}\right)\right)=\left(H\left(\left(X_{i}, Y_{i}\right),\left(X_{j}, Y_{j}\right)\right)+H\left(\left(X_{j}, Y_{j}\right),\left(X_{i}, Y_{i}\right)\right)\right) / 2$ is a symetric kernel. Hence, $V_{n}$ is by definition (see, e.g., Serfling, 1980, p. 174) the Von Mises statistic associated to the parametric function

$$
\begin{aligned}
\theta(F) & =E_{F}\left[\mathcal{H}\left(\left(X_{1}, Y_{1}\right),\left(X_{2}, Y_{2}\right)\right)\right] \\
& =\left[E_{F} H\left(\left(X_{1}, Y_{1}\right),\left(X_{2}, Y_{2}\right)\right)+E_{F} H\left(\left(X_{2}, Y_{2}\right),\left(X_{1}, Y_{1}\right)\right)\right] / 2 .
\end{aligned}
$$

Let $H_{1}\left(X_{1}, Y_{1}\right)=E_{F}\left[H\left(\left(X_{1}, Y_{1}\right),\left(X_{2}, Y_{2}\right)\right) \mid\left(X_{1}, Y_{1}\right)\right]$. For any $\left(x_{1}, y_{1}\right)$, we have $H_{1}\left(x_{1}, y_{1}\right)=$ $E_{F}\left[H\left(\left(x_{1}, y_{1}\right),\left(X_{2}, Y_{2}\right)\right)\right]=0$. Then $H_{1}\left(X_{1}, Y_{1}\right)=0$, whence $E_{F}\left[H_{1}\left(X_{1}, Y_{1}\right)\right]=0$, i.e., $E_{F}\left[H\left(\left(X_{1}, Y_{1}\right),\left(X_{2}, Y_{2}\right)\right)\right]=0$. We obtain in a similar way $E_{F}\left[H\left(\left(X_{2}, Y_{2}\right),\left(X_{1}, Y_{1}\right)\right)\right]=0$, which gives $\theta(F)=0$. Putting $W_{n}=\frac{1}{n^{2}} \sum_{i=1}^{n} \sum_{j=1}^{n} H\left(\left(X_{i}, Y_{i}\right),\left(X_{j}, Y_{j}\right)\right)$, we get via Theorem A and Lemma 5.7.3 of Serfling (1980, p. 192 and 206) that $\sqrt{n} W_{n} \stackrel{\mathcal{L}}{\longrightarrow} N\left(0,4 \zeta_{1}\right)$, where

$$
\begin{aligned}
\zeta_{1} & =\operatorname{Var}_{F}\left\{E_{F}\left[\mathcal{H}\left(\left(X_{1}, Y_{1}\right),\left(X_{2}, Y_{2}\right)\right) \mid\left(X_{1}, Y_{1}\right)\right]\right\} \\
& =\operatorname{Var}_{F}\left\{E_{F}\left[H\left(\left(X_{2}, Y_{2}\right),\left(X_{1}, Y_{1}\right)\right) \mid\left(X_{1}, Y_{1}\right)\right]\right\} / 4
\end{aligned}
$$

This last result follows from the fact that $H_{1}\left(X_{1}, Y_{1}\right)=0$. On the other hand, if the conditions of Theorem 2.1 hold, then $\sup _{x \in K} \sqrt{n}\left|R_{n}^{\alpha}(x)\right| \stackrel{P}{\longrightarrow} 0$ by Corollary 2.1. If furthermore the scalar functions $g_{j}(\cdot)$ are bounded on $K$, then the vector $\frac{1}{n} \sum_{i=1}^{n} \mathbb{1}_{K}\left(X_{i}\right) g\left(X_{i}\right)\left\{\sqrt{n} R_{n}^{\alpha}\left(X_{i}\right)\right\}$ converges in probability to 0 as $n \rightarrow \infty$. Finally, since $\left\{\frac{1}{n} \sum_{i=1}^{n} \mathbb{1}_{K}\left(X_{i}\right) g\left(X_{i}\right) g^{T}\left(X_{i}\right)\right\}^{-1}$ converges to $\left\{E\left[\mathbb{1}_{K}\left(X_{1}\right) g\left(X_{1}\right) g^{T}\left(X_{1}\right)\right]\right\}^{-1}$ in probability via the law of large numbers, we get the desired conclusion from the following representation

$$
\begin{aligned}
\sqrt{n}\left(\widehat{\theta}_{\alpha, n}\left(K, \widehat{\mathbb{P}}_{X, n}\right)-\theta_{\alpha}\left(K, \widehat{\mathbb{P}}_{X, n}\right)\right)=\sqrt{n} V_{n}\left\{\frac{1}{n} \sum_{i=1}^{n} \mathbb{1}_{K}\left(X_{i}\right) g\left(X_{i}\right) g^{T}\left(X_{i}\right)\right\}^{-1} \\
=\left[\sqrt{n} W_{n}+\frac{1}{n} \sum_{i=1}^{n} \mathbb{1}_{K}\left(X_{i}\right) g\left(X_{i}\right)\left\{\sqrt{n} R_{n}^{\alpha}\left(X_{i}\right)\right\}\right]\left\{\frac{1}{n} \sum_{i=1}^{n} \mathbb{1}_{K}\left(X_{i}\right) g\left(X_{i}\right) g^{T}\left(X_{i}\right)\right\}^{-1} .
\end{aligned}
$$

\section{A.4 Proof of Theorem 3.2}

We have in view of the first order conditions which define $\widehat{\theta}_{\alpha, n}(K, \mu)$,

$$
\left.\int_{K}\left(\widehat{q}_{\alpha, n}(x)-\varphi_{\widehat{\theta}_{\alpha, n}(K, \mu)}(x)\right) \frac{\partial \varphi_{\theta}}{\partial \theta}(x)\right|_{\theta=\widehat{\theta}_{\alpha, n}(K, \mu)} d \mu(x)=0 .
$$

It follows

$$
\begin{aligned}
& \left.\sqrt{n} \int_{K} \frac{\partial \varphi_{\theta}}{\partial \theta}(x)\right|_{\theta=\widehat{\theta}_{\alpha, n}(K, \mu)}\left(\widehat{q}_{\alpha, n}(x)-q_{\alpha}(x)\right) d \mu(x) \\
& +\left.\sqrt{n} \int_{K} \frac{\partial \varphi_{\theta}}{\partial \theta}(x)\right|_{\theta=\widehat{\theta}_{\alpha, n}(K, \mu)}\left(q_{\alpha}(x)-\varphi_{\theta_{\alpha}(K, \mu)}(x)\right) d \mu(x) \\
& =\left.\sqrt{n} \int_{K} \frac{\partial \varphi_{\theta}}{\partial \theta}(x)\right|_{\theta=\widehat{\theta}_{\alpha, n}(K, \mu)}\left(\varphi_{\widehat{\theta}_{\alpha, n}(K, \mu)}(x)-\varphi_{\theta_{\alpha}(K, \mu)}(x)\right) d \mu(x) .
\end{aligned}
$$


In this equality, $\left.\frac{\partial \varphi_{\theta}}{\partial \theta}(x)\right|_{\theta=\widehat{\theta}_{\alpha, n}(K, \mu)}$ can be replaced by $\left.\frac{\partial \varphi_{\theta}}{\partial \theta}(x)\right|_{\theta=\theta_{\alpha}(K, \mu)}$ and we note that the second term on the left hand side is then equal to zero, by the definition of $\theta_{\alpha}(K, \mu)$. By Young's form of Taylor's expansion of the first order, we have for any $x \in K$,

$$
\begin{aligned}
\varphi_{\widehat{\theta}_{\alpha, n}(K, \mu)}(x)-\varphi_{\theta_{\alpha}(K, \mu)}(x)= & \left.\frac{\partial \varphi_{\theta}}{\partial \theta^{T}}(x)\right|_{\theta=\theta_{\alpha}(K, \mu)}\left(\widehat{\theta}_{\alpha, n}(K, \mu)-\theta_{\alpha}(K, \mu)\right) \\
& +\varepsilon\left(\widehat{\theta}_{\alpha, n}(K, \mu)-\theta_{\alpha}(K, \mu)\right)\left(\widehat{\theta}_{\alpha, n}(K, \mu)-\theta_{\alpha}(K, \mu)\right)
\end{aligned}
$$

where $\varepsilon\left(\widehat{\theta}_{\alpha, n}(K, \mu)-\theta_{\alpha}(K, \mu)\right)=o_{p}(1)$ as $n \rightarrow \infty$, uniformly in $x \in K$, since $\widehat{\theta}_{\alpha, n}(K, \mu)-$ $\theta_{\alpha}(K, \mu) \stackrel{P}{\longrightarrow} 0$ and the differentiability of $\theta \mapsto \varphi_{\theta}(x)$ at $\theta_{\alpha}(K, \mu)$ is uniform in $x \in K$. Therefore

$$
\begin{aligned}
& \left.\sqrt{n} \int_{K} \frac{\partial \varphi_{\theta}}{\partial \theta}(x)\right|_{\theta=\theta_{\alpha}(K, \mu)}\left(\varphi_{\hat{\theta}_{\alpha, n}(K, \mu)}(x)-\varphi_{\theta_{\alpha}(K, \mu)}(x)\right) d \mu(x) \\
& =\left(\left.\left.\int_{K} \frac{\partial \varphi_{\theta}}{\partial \theta}(x)\right|_{\theta=\theta_{\alpha}(K, \mu)} \frac{\partial \varphi_{\theta}}{\partial \theta^{T}}(x)\right|_{\theta=\theta_{\alpha}(K, \mu)} d \mu(x)\right)\left\{\sqrt{n}\left(\widehat{\theta}_{\alpha, n}(K, \mu)-\theta_{\alpha}(K, \mu)\right)\right\} \\
& +\left(\left.\int_{K} \frac{\partial \varphi_{\theta}}{\partial \theta}(x)\right|_{\theta=\theta_{\alpha}(K, \mu)} d \mu(x)\right) \varepsilon\left(\widehat{\theta}_{\alpha, n}(K, \mu)-\theta_{\alpha}(K, \mu)\right)\left\{\sqrt{n}\left(\widehat{\theta}_{\alpha, n}(K, \mu)-\theta_{\alpha}(K, \mu)\right)\right\} .
\end{aligned}
$$

It follows

$$
\begin{aligned}
\sqrt{n}\left(\widehat{\theta}_{\alpha, n}(K, \mu)-\theta_{\alpha}(K, \mu)\right)=\{ & \left.\left.\int_{K} \frac{\partial \varphi_{\theta}}{\partial \theta^{T}}(x)\right|_{\theta=\theta_{\alpha}(K, \mu)} \frac{\partial \varphi_{\theta}}{\partial \theta}(x)\right|_{\theta=\theta_{\alpha}(K, \mu)} d \mu(x) \\
& \left.+\left.\int_{K} \frac{\partial \varphi_{\theta}}{\partial \theta^{T}}(x)\right|_{\theta=\theta_{\alpha}(K, \mu)} d \mu(x) \varepsilon\left(\widehat{\theta}_{\alpha, n}(K, \mu)-\theta_{\alpha}(K, \mu)\right)\right\}^{-1} \\
& \times\left.\int_{K} \frac{\partial \varphi_{\theta}}{\partial \theta}(x)\right|_{\theta=\theta_{\alpha}(K, \mu)}\left\{\sqrt{n}\left(\widehat{q}_{\alpha, n}(x)-q_{\alpha}(x)\right)\right\} d \mu(x) .
\end{aligned}
$$

We finally obtain the desired convergence by using Theorem 2.1 .

\section{A.5 Proof of Theorem 3.3}

Putting $M_{n}(\theta)=\int_{K}\left(\widehat{\varphi}_{n}(x)-\varphi_{\theta}(x)\right)^{2} d \mu(x)$, it can be easily seen that

$$
\sup _{\theta \in \mathbb{R}^{k}}\left|M_{n}(\theta)-M(\theta)\right| \leq 2(\nu+\delta) \mu(K) \sup _{x \in K}\left|\widehat{\varphi}_{n}(x)-\varphi(x)\right|
$$

with probability 1. Then $\sup _{\theta \in \mathbb{R}^{k}}\left|M_{n}(\theta)-M(\theta)\right| \stackrel{\text { a.s. }}{\rightarrow} 0$ since $\sup _{x \in K}\left|\widehat{\varphi}_{n}(x)-\varphi(x)\right| \stackrel{a . s .}{\rightarrow} 0$ by Lemma 3.3 in Daouia and Simar (2005). Whence $Z_{n}:=M_{n}(\theta(K, \mu))-M(\theta(K, \mu)) \stackrel{a . s .}{\rightarrow} 0$. Since $M_{n}\left(\widehat{\theta}_{n}(K, \mu)\right) \leq M_{n}(\theta(K, \mu))$ by definition of $\widehat{\theta}_{n}(K, \mu)$, we obtain $M_{n}\left(\widehat{\theta}_{n}(K, \mu)\right) \leq$ $M(\theta(K, \mu))+Z_{n}$. Therefore

$$
\begin{aligned}
0 \leq M\left(\widehat{\theta}_{n}(K, \mu)\right)-M(\theta(K, \mu)) & \leq M\left(\widehat{\theta}_{n}(K, \mu)\right)-M_{n}\left(\widehat{\theta}_{n}(K, \mu)\right)+Z_{n} \\
& \leq \sup _{\theta \in \mathbb{R}^{k}}\left|M_{n}(\theta)-M(\theta)\right|+Z_{n} \stackrel{a . s}{\longrightarrow} 0 .
\end{aligned}
$$


Thus $M\left(\widehat{\theta}_{n}(K, \mu)\right) \stackrel{\text { a.s. }}{\longrightarrow} M(\theta(K, \mu))$, which is equivalent to $\lim _{n \rightarrow \infty} P\left[\sup _{m \geq n} \mid M\left(\widehat{\theta}_{m}(K, \mu)\right)-\right.$ $M(\theta(K, \mu)) \mid>\eta]=0$ for every $\eta>0$. Let us now show that $\widehat{\theta}_{n}(K, \mu) \stackrel{a . s .}{\longrightarrow} \theta(K, \mu)$, which is equivalent to show that $\lim _{n \rightarrow \infty} P\left[\sup _{m \geq n} d\left(\widehat{\theta}_{m}(K, \mu), \theta(K, \mu)\right)>\varepsilon\right]=0$, for every $\varepsilon>0$. Because $\inf _{d(\theta, \theta(K, \mu)) \geq \varepsilon} M(\theta)>M(\theta(K, \mu))$, there exists a number $\eta_{\varepsilon}>0$ such that $\inf _{d(\theta, \theta(K, \mu)) \geq \varepsilon} M(\theta)>M(\theta(K, \mu))+\eta_{\varepsilon}$. It follows that the event $\left\{d\left(\widehat{\theta}_{n}(K, \mu), \theta(K, \mu)\right) \geq \varepsilon\right\}$ is contained in $\left\{M\left(\widehat{\theta}_{n}(K, \mu)\right)>M(\theta(K, \mu))+\eta_{\varepsilon}\right\}$, for all $n \geq 1$. Hence it can be easily seen that $\left\{\sup _{m \geq n} d\left(\widehat{\theta}_{m}(K, \mu), \theta(K, \mu)\right)>\varepsilon\right\}$ is contained in the event $\left\{\sup _{m \geq n} \mid M\left(\widehat{\theta}_{m}(K, \mu)\right)-\right.$ $\left.M(\theta(K, \mu)) \mid>\eta_{\varepsilon}\right\}$, which ends the proof.

\section{A.6 Proof of Theorem 3.4}

For any $\varepsilon>0$ and any $\eta_{\varepsilon} \in\left(0, m_{\varepsilon}\right)$ we have $\inf _{d(\theta, \theta(K, \mu)) \geq \varepsilon} M(\theta)>M(\theta(K, \mu))+\eta_{\varepsilon}$ in view of (3.7). It follows that the event $\left\{d\left(\widehat{\theta}_{m(n), n}(K, \mu), \theta(K, \mu)\right) \geq \varepsilon\right\}$ is contained in $\left\{M\left(\widehat{\theta}_{m(n), n}(K, \mu)\right)>M(\theta(K, \mu))+\eta_{\varepsilon}\right\}$, for all $n \geq 1$. Putting

$$
M_{m(n)}(\theta)=\int_{K}\left(\widehat{\varphi}_{m(n), n}(x)-\varphi_{\theta}(x)\right)^{2} d \mu(x)
$$

we show in the same way as in (A.14) that

$$
M\left(\widehat{\theta}_{m(n), n}(K, \mu)\right)-M(\theta(K, \mu)) \leq 2 \sup _{\theta \in \mathbb{R}^{k}}\left|M_{m(n)}(\theta)-M(\theta)\right| .
$$

Hence, we obtain for all $n \geq 1$,

$$
P\left[d\left(\widehat{\theta}_{m(n), n}(K, \mu), \theta(K, \mu)\right) \geq \varepsilon\right] \leq P\left[\sup _{\theta \in \mathbb{R}^{k}}\left|M_{m(n)}(\theta)-M(\theta)\right|>\eta_{\varepsilon} / 2\right] .
$$

On the other hand, we have with probability 1 ,

$$
\sup _{\theta \in \mathbb{R}^{k}}\left|M_{m(n)}(\theta)-M(\theta)\right| \leq 2(\nu+\delta) \mu(K) \sup _{x \in K}\left|\widehat{\varphi}_{m(n), n}(x)-\varphi(x)\right| .
$$

Therefore, for any $\varepsilon>0$, any $\eta_{\varepsilon} \in\left(0, m_{\varepsilon}\right)$ and all $n \geq 1$, we have

$$
P\left[d\left(\widehat{\theta}_{m(n), n}(K, \mu), \theta(K, \mu)\right) \geq \varepsilon\right] \leq P\left[\sup _{x \in K}\left|\widehat{\varphi}_{m(n), n}(x)-\varphi(x)\right|>\eta_{\varepsilon} /(4(\nu+\delta) \mu(K))\right] .
$$

Thus, the first inequality (3.8) follows immediately from Corollary 2.4 in Daouia and Simar (2005) by putting $\eta_{\varepsilon} /(4(\nu+\delta) \mu(K))=\lambda r$. We show the second inequality (3.9) by using the same arguments in conjunction with Corollary 2.5 in Daouia and Simar (2005). 


\section{References}

[1] Aigner, D.J. and S.F. Chu (1968), On estimating the industry production function, American Economic Review, 58, 826-839.

[2] Aragon, Y. and Daouia, A. and Thomas-Agnan, C. (2005), Nonparametric Frontier Estimation: A Conditional Quantile-based Approach, Econometric Theory, 21, 358-389.

[3] Cazals, C., J.P. Florens and L. Simar (2002), Nonparametric frontier estimation: a robust approach, Journal of Econometrics, 106, 1-25.

[4] Daouia, A. (2005), Asymptotic Representation Theory for Nonstandard Conditional Quantiles, Journal of Nonparametric Statistics, 17(2), 253-268.

[5] Daouia, A. and Ruiz-Gazen, A. (2006), Robust Nonparametric Frontier Estimators: Influence Function and Qualitative Robustness, Statistica Sinica, 16 (4), 1233-1253.

[6] Daouia, A. and L. Simar (2005), Robust Nonparametric Estimators of Monotone Boundaries, Journal of Multivariate Analysis, 96, 311-331.

[7] Daouia, A. and L. Simar (2006), Nonparametric efficiency analysis: a multivariate conditional quantile approach, Discussion paper 0419, Institut de Statistique, UCL, forthcoming in Journal of Econometrics.

[8] Deprins, D., Simar, L. and H. Tulkens (1984), Measuring labor inefficiency in post offices. In The Performance of Public Enterprises: Concepts and measurements. M. Marchand, P. Pestieau and H. Tulkens (eds.), Amsterdam, North-Holland, 243-267.

[9] Florens, J.P. and L. Simar, (2005), Parametric Approximations of Nonparametric Frontier, Journal of Econometrics, 124 (1), 91-116.

[10] Greene, W. H. (1980), Maximum likelihood estimation of econometric frontier functions, Journal of Econometrics, 13 (1), 27-56.

[11] Korostelev, A., Simar, L. and A. Tsybakov (1995), Efficient Estimation of Monotone Boundaries, Annals of Statistics, 23(2), 476-489.

[12] Lovell, C. A. K. (1993), "Production Frontiers and Productive Efficiency," in The Measurement of Productive Efficiency: Techniques and Applications, ed. by Hal Fried, C. A. Knox Lovell, and Shelton S. Schmidt, Oxford University Press, Oxford, pp. 3-67. 
[13] Markovitz, H. M. (1959), Portfolio Selection: Efficient Diversification of Investments. John Wiley, New York.

[14] Mouchart, M. and L. Simar (2002), Efficiency analysis of Air Controlers: first insights, Consulting report 0202, Institut de Statistique, Université Catholique de Louvain, Belgium.

[15] Park, B. Simar, L. and Ch. Weiner (2000), The FDH Estimator for Productivity Efficiency Scores : Asymptotic Properties, Econometric Theory, Vol 16, 855-877.

[16] Seiford, L.M. (1996), Data envelopment analysis: The evolution of the state-of-the-art (1978-1995), Journal of Productivity Analysis, 7, 2/3, 99-138.

[17] Sengupta, J. K. (1991), Maximum probability dominance and portfolio theory, J.Optim. Theory Appl. 71, 341-357.

[18] Sengupta, J. K., and Park, H.S. (1993), Portfolio efficiency tests based on stochastic dominance and cointegration, Internat. J. Systems Sci. 24, 2135-2158.

[19] Serfling, R. J. (1980), Approximation Theorems of Mathematical Statistics, John Wiley, New York.

[20] Simar, L. (1992), Estimating efficiencies from frontier models with panel data: a comparison of parametric, non-parametric and semi-parametric methods with bootstrapping, Journal of Productivity Analysis, 3, 167-203.

[21] van der Vaart, A. W. (1998) Asymptotic Statistics. Cambridge Series in Statistical and Probabilistic Mathematics, 3, Cambridge University Press, Cambridge.

[22] van der Vaart, A. W. and J.A. Wellner, (1996) Weak Convergence and Empirical Processes. With Applications to Statistics. Springer Series in Statistics, Springer-Verlag, New York. 


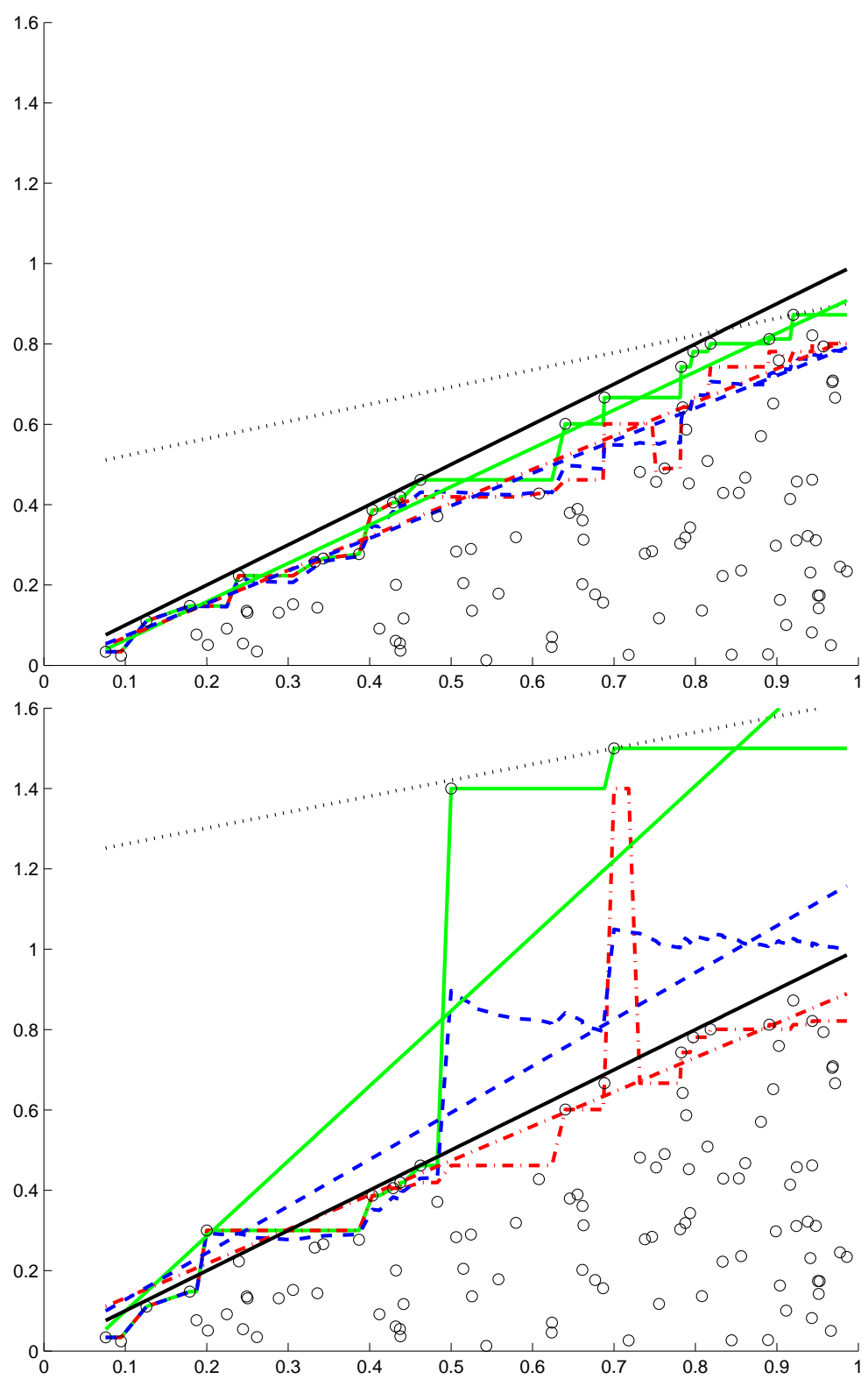

Figure 1: Results for Example 1. In solid black line, the true frontier $y=x$. In cyan solid, the FDH frontier estimate and its corresponding parametric fit, in blue dashed the order-m frontier $\widehat{\varphi}_{m, n}$, and its corresponding parametric fit and in dash-dot red the order- $\alpha$ frontier $\widehat{q}_{\alpha, n}$ and its corresponding parametric fit. Here, $m=20$ and $\alpha=0.9622$. From top to bottom 100 simulated observations without outliers, 100 simulated observations with 3 outliers included. In black dotted, the shifted OLS estimate. 


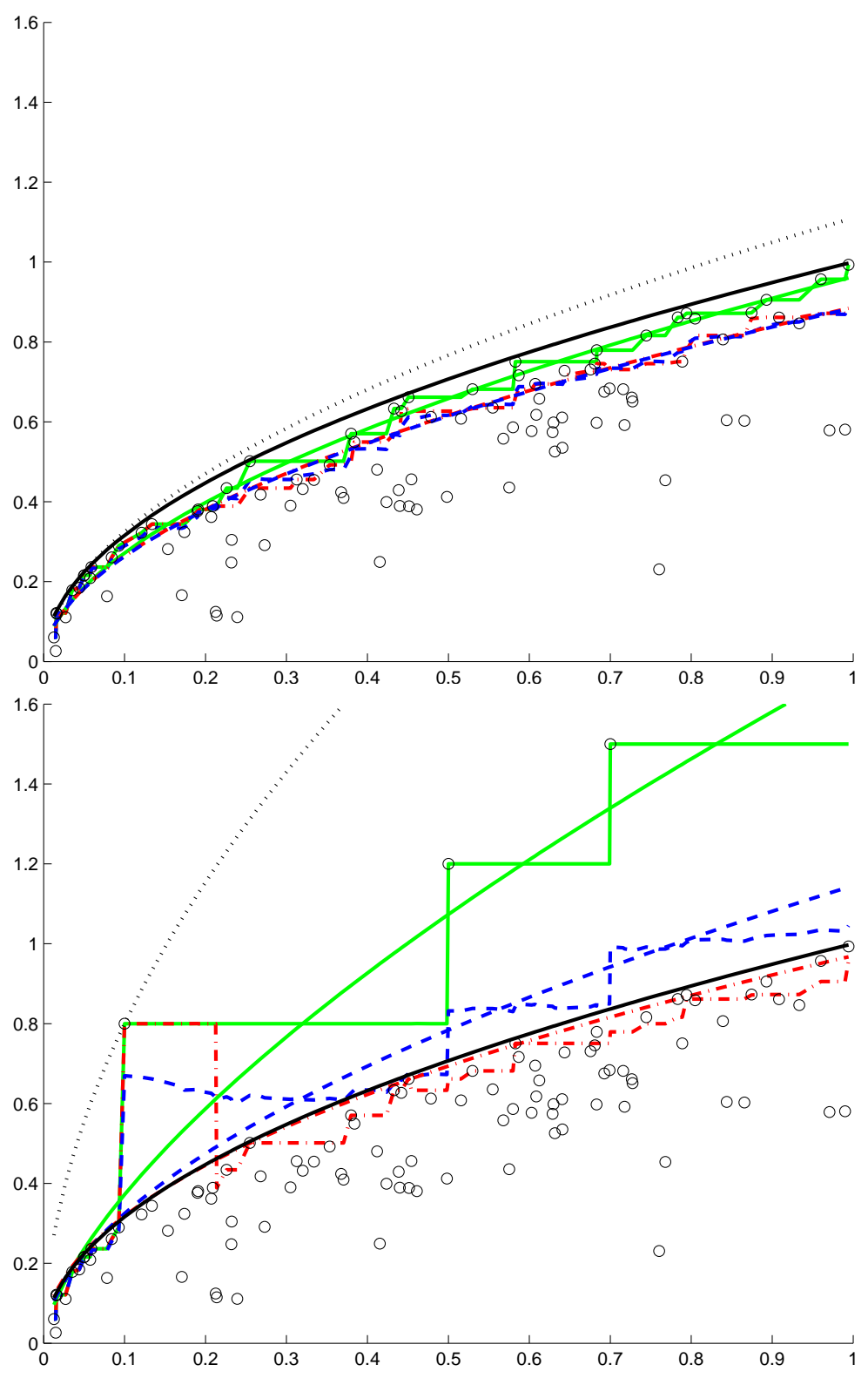

Figure 2: Results for Example 2. In solid black line, the true frontier $y=x^{0.5}$. In cyan solid, the FDH frontier estimate and its corresponding parametric fit, in blue dashed the order-m frontier $\widehat{\varphi}_{m, n}$, and its corresponding parametric fit and in dash-dot red the order- $\alpha$ frontier $\widehat{q}_{\alpha, n}$ and its corresponding parametric fit. Here, $m=20$ and $\alpha=0.9612$. From top to bottom 100 simulated observations without outliers, 100 simulated observations with 3 outliers included. In black dotted, the shifted OLS estimate. 


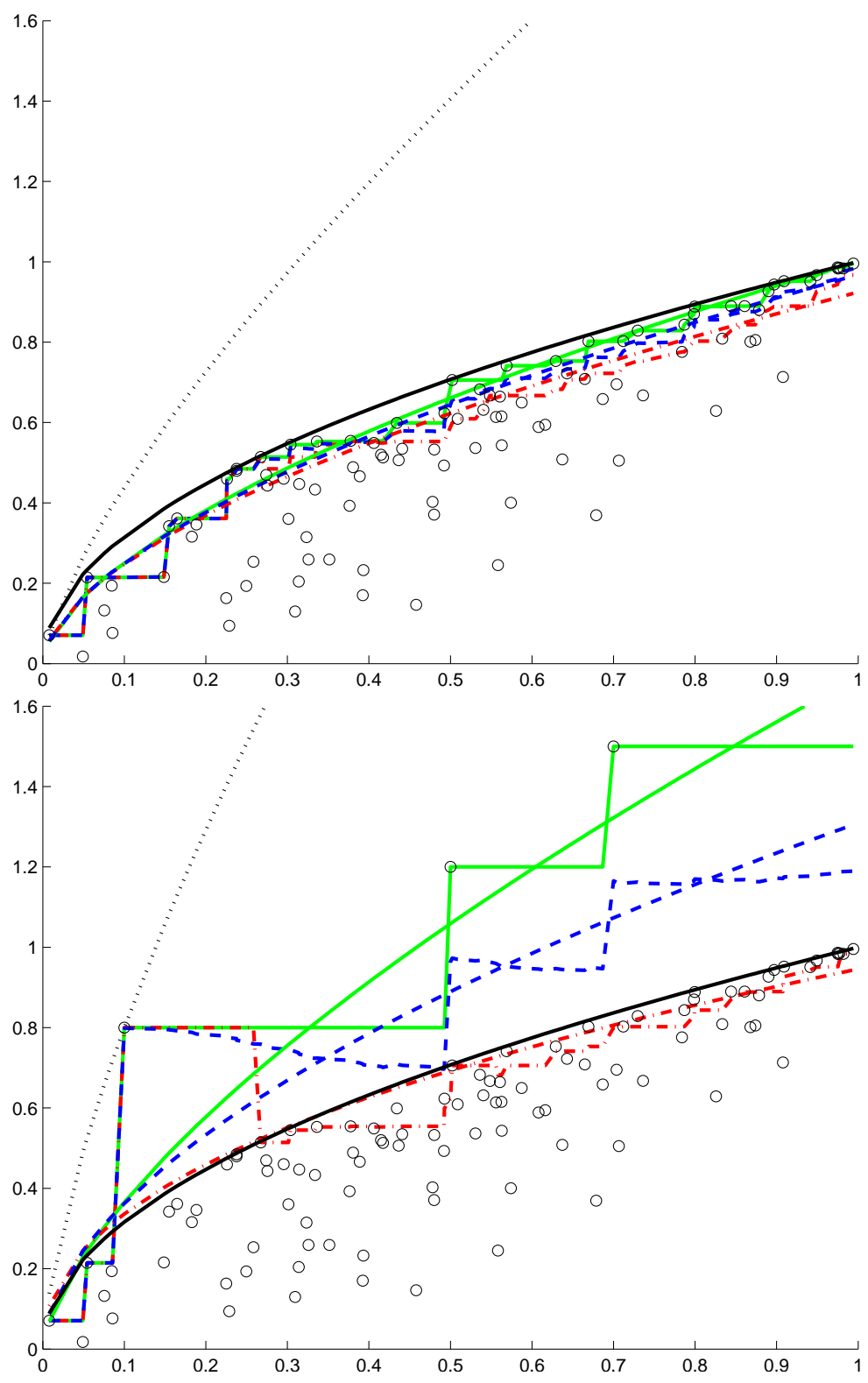

Figure 3: Results for Example 3. In solid black line, the true frontier $y=x^{0.5}$. In cyan solid, the FDH frontier estimate and its corresponding parametric fit, in blue dashed the order-m frontier $\widehat{\varphi}_{m, n}$, and its corresponding parametric fit and in dash-dot red the order$\alpha$ frontier $\widehat{q}_{\alpha, n}$ and its corresponding parametric fit. Here, $m=38$ and $\alpha=0.95$. From top to bottom 100 simulated observations without outliers, 100 simulated observations with 3 outliers included. In black dotted, the shifted OLS estimate. 


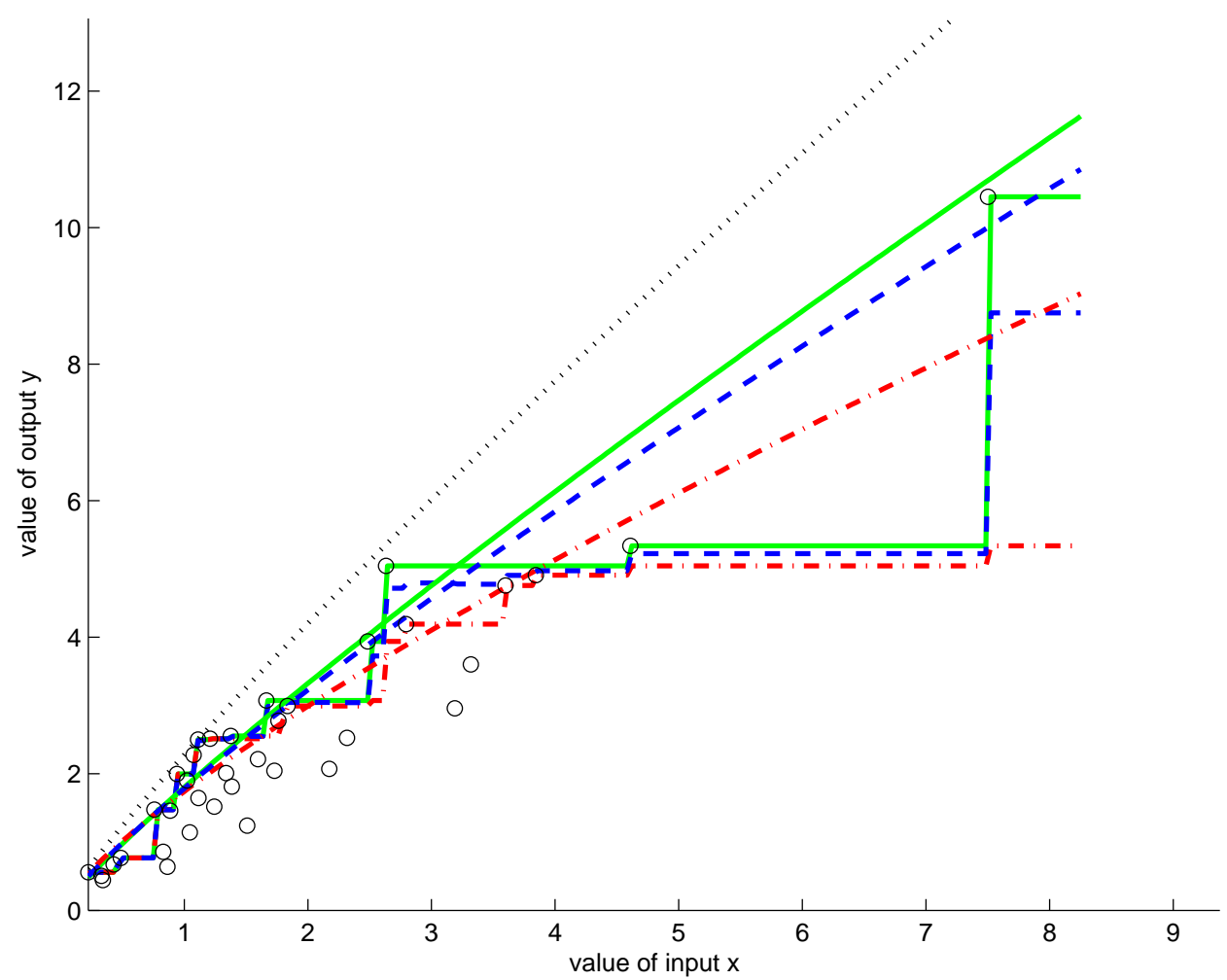

Figure 4: Air controlers example. In cyan solid, the FDH frontier estimate and its corresponding parametric fit, in blue dashed the order- $m$ frontier $\widehat{\varphi}_{m, n}$, and its corresponding parametric fit and in dash-dot red the order- $\alpha$ frontier $\widehat{q}_{\alpha, n}$ and its corresponding parametric fit. Here, $m=41$ and $\alpha=0.95$. In black dotted, the shifted OLS estimate. 